\title{
The Law of Conservation of Time and Its Applications
}

\author{
Ninh Khac Son \\ Email: alodoanhnhan@gmail.com \\ Date Performed: 01 December 2020
}

\begin{abstract}
Time is a complex category not only in philosophy but also in mathematics and physics. In one thought about time, the author accidentally discovered a new way to explain and solve problems related to time dilation, such as solving the problem of Muon particle when moving from a height of $10 \mathrm{~km}$ to the earth's surface, while the Muon's lifespan is only 2.2 microseconds, or explaining Michelson-Morley experiment using the new method.

In addition, the author also prove that the speed of light in vacuum is the maximum speed in the universe, and discovered the red shift effect while there is no increase in distance between objects. To do this, the author has built two axioms based on the discontinuity in the motion of the object and draw two consequences along with the law of conservation of time.
\end{abstract}

Key words: The law of conservation of time; time dilation; red shift; Michelson-Morley experiment; maximum speed; formation of radiation; subatomic particle disappears.

\section{The Law of Conservation of Time}

\subsection{Method of determining the intermittent motion of the object.}

Intermittent motion is defined as the existence of a rest periods in the motion of the object, for example if object $\mathrm{A}$ moves in time $T_{1}$ and rest in time $T_{2}$, then continues in motion in time $T_{3}$. We now call the motion of $\mathrm{A}$ in time $\mathrm{T}$ the intermittent motion: $T=T_{1}+T_{2}+T_{3}$

In contrast, continuous motion is understood as there is no immobility time in the motion of the object. 
If we use the above method to determine the intermittent motion then we need to observe the entire motion of the object to find the immobility time. This method has limitations in cases where the very small(almost imperceptible) immobility time is interlaced and "smoothed" with the motion time of the object.

For example: is the motion of electrons, quarks, light, ... intermittent motion or continuous motion? We cannot apply the above method to solve the problem.

Therefore, we need to build a more complete method to determine intermittent motion. This is a method based only on the comparison of speed (fast, slow, velocity) between objects and then derive the results, the method is presented as follows:

Case 1:

Assume the motion of two objects $\mathrm{A}$ and $\mathrm{B}$ are the intermittent motions, where the velocity of $\mathrm{A}$ is greater than $\mathrm{B}\left(v_{A}>v_{B}\right)$.Then we explain the velocity difference of $\mathrm{A}$ and $\mathrm{B}$ through the intermittent motion as follows:

- The velocity of $A$ is greater than $B$ because $A$ moves intermittently with less immobility time than object $B$, so $A$ moves faster than $B$.

- Analyzing in the opposite direction, we have the velocity of $B$ less than $A$ because $B$ moves intermittently with more immobility time than $A$, so $B$ moves more slowly than $A$.

- In addition, we have one more explanation: $v_{B}<v_{A}$ because $B$ moves more intermittently than $A$; otherwise $v_{A}>v_{B}$ because $A$ moves less intermittently than $B$.

\section{Comment in case 1:}

When the velocity is explained through the intermittent motion of the objects then they produce meaningful and easily accepted content, hence the explanation of velocity through intermittent motion is acceptable and logical.

Case 2:

Assume the motion of two objects $\mathrm{C}$ and $\mathrm{D}$ are the continuous motions, where 
the velocity of $\mathrm{C}$ is greater than $\mathrm{D}\left(v_{C}>v_{D}\right)$. Then we explain the velocity difference of $\mathrm{C}$ and $\mathrm{D}$ through the continuous motion as follows:

- The velocity of $C$ is greater than $D$ because $C$ moves continuously with motion time more than $D$, so $C$ moves faster than $D$.

- Analyzing in the opposite direction, we have the velocity of D less than $C$ because $D$ moves continuously with less motion time than object $C$, so $D$ moves more slowly than $C$.

- In addition, we have one more explanation: $v_{C}>v_{D}$ because $C$ moves more continuously than $D$; otherwise $v_{D}<v_{C}$ because $D$ moves less continuously than $C$.

Comment in case 2:

- The interpretation of velocity through continuous motion is not satisfactory, because the motion time of $D$ less than $C$ means there is still immobility time in the motion of $D$, this implies that the motion of $D$ is an intermittent motion and contradicts the hypothesis.

- In addition, explanation is also not satisfactory if based on " $v_{C}>v_{D}$ because $C$ moves more continuous than D". Because continuity is perfect and the term "more continuous" doesn't exist, the perfection of continuity is like that of the perfection the circle or square, there is no more rounded shape than circle or square than square.

Construct the axiom through two cases:

For the velocity explanations through continuous motion, we cannot enumerate all the explanations, so we cannot negate all of them.

But based on the arguments that we have, we construct an axiom that is the basis for determining the intermittent motion of the object as follows:

Statement of the first axiom:

\section{"The motion of object $A$ is defined as the intermittent motion in time $\mathrm{T}$ if and only if $\mathrm{A}$ moves at a velocity less than that of object $\mathrm{B}$ in time $\mathrm{T}$ ".}

$\mathrm{B}$ is any object, just need to determine that the velocity of $\mathrm{B}$ is greater than A then we conclude: "Motion of A is the intermittent motion" - That is the content and meaning of the axiom. 
Note:

The axiom is built as a foundation to draw consequences and laws, the methods of proving the consequences and the law are also based on this axiom. Therefore, the axiom is crucial to the entire content of the manuscript.

\subsection{Method of determining the continuous motion of the object.}

In section 1.1, we constructed the axiom to determine the intermittent motion of object A, for example the motion of the following objects is the intermittent motion:

- The velocity of bicycle $A$ is less than that of car B, so the motion of bicycle $A$ is the intermittent motion.

- The velocity of car $B$ is less than that of airplane $C$, so the motion of car $B$ is the intermittent motion.

- The velocity of plane $C$ is less than that of rocket $D$, so the motion of plane $C$ is the intermittent motion.

$\cdots$

If we determine continuous motion using the method listed above then we cannot find a result. Because even if we can determine that the speed of light is the maximum speed, it is not guaranteed that the motion of light is the continuous

Therefore, we need to construct a consequence of continuous motion based on the given axiom.

Statement of consequence:

"The motion of object $\mathrm{A}$ in time $\mathrm{T}$ is the continuous motion if and only if A moves at maximum speed $C_{\max }$ in the universe in time T".

In which $C_{\max }$ is built as the maximum velocity in the universe, no object has a speed greater than $C_{\max }$. And when we have not found the real value of $C_{\max }$, we still show the independence of $C_{\max }$ when assigning the value: $C_{\max } \geq c=299,792,458 \mathrm{~m} / \mathrm{s}$.

Prove the consequence: 
Prove the first side: If the object $\mathrm{A}$ is continuously moving then $\mathrm{A}$ is moving at maximum speed $C_{\max }$ in the universe.

Let $\mathrm{A}$ be the continuously moving object corresponding to $v_{A}$, and $\mathrm{B}$ be the moving object with the velocity of $v_{B}$. Because motion of $\mathrm{A}$ is the continuous motion, so the velocity of $\mathrm{A}$ is always greater than or equal to $\mathrm{B}$ in all cases corresponding to the velocity of $\mathrm{B}$.

We have:

$$
\begin{aligned}
& v_{A} \geq v_{B}, \forall v_{B} \\
& \rightarrow v_{A}-\lim _{v_{B} \rightarrow C_{\max }} \geq 0 \\
& \rightarrow v_{A}=C_{\max }
\end{aligned}
$$

Prove the second side: If A moves at maximum speed in the universe then the motion of $\mathrm{A}$ is the continuous

According to the given assumption, $C_{\max }$ is the maximum velocity in the universe and no object moves at a faster speed than $C_{\max }$. Therefore, the motion of $\mathrm{A}$ at $C_{\max }$ in time $\mathrm{T}$ does not contain immobility time, because if there exists a immobility time in the motion of $\mathrm{A}$ in time $\mathrm{T}$ then $C_{\max }$ is not the maximum speed.

And since motion of $\mathrm{A}$ in time $\mathrm{T}$ contains no immobility time, so motion of $\mathrm{A}$ is a continuous motion in time $\mathrm{T}$.

Proving end.

\subsection{Matter is always resting in space.}

We found no evidence that objects made of matter travel at an average speed equal to the speed of light in a vacuum. In other words: the speed of light in a vacuum is the limited inaccessible speed of all matter objects.

But this is not provable, because experimental results are never enough, experimental results can only be evidence but not the basis of proving methods. 
Therefore, we need to build a second axiom based on these experimental evidence.

Statement of the second axiom:

"The average velocity of matter in time $\mathrm{T}$ is always less than the maximum velocity $C_{\max }$ in the universe."

Because $C_{\max }$ is built by us to have $C_{\max } \geq c$ value, so if we cannot find evidence that matter moves at speed c then the average velocity of matter is less than $C_{\max }$.

The axiom also contains two important meanings:

- If the velocity of an object in time $T$ is valid $v \in\left[c, C_{\max }\right]$ then that object is not matter.

- The motion of all matter in time $T$ is the intermittent motion.

On the other hand, we know that the intermittent motion of the object in time $\mathrm{T}$ is the motion in which there is a intertwining of immobility time and the motion time of the object in time T. The intertwining of immobility time and motion time is visible to the naked eye in some cases, but almost impossible to observe with the naked eye in most cases of motion in nature.

For example:

An electron moves at constant velocity in time $T$, then the velocity $v_{e}$ of the electron is calculated using the following expression:

$$
v_{e}=\frac{\lambda}{T_{\text {cycle }}}=\frac{S}{T}
$$

Where:

- $v_{e}$ is the velocity of the electron.

- $\lambda$ is the wavelength of the electron.

- $S$ is the distance traveled by the electron in time $T$.

- $T_{\text {cycle }}$ is the time of one cycle of the electron.

Thus, the value $v_{e}$ was calculated in one cycle $T_{\text {cycle, }}$, so the resting time existed 
within one cycle $T_{\text {cycle. }}$ Therefore, if we consider the whole time $T=n * T_{\text {cycle }}$ then the immobility time and motion time are intertwined in a regular and complex way that the naked eye cannot recognize.

We construct the consequence to describe the intertwining of immobility time and motion time in the intermittent motion of the object as follows:

Statement of consequence:

"The intermittent motion of an object in time $T$ is an intertwining process between the immobility time and the motion time of the object in time $\mathrm{T}$, in which the motion time corresponds to the object is moving at $C_{\max }$ during that time."

Prove the consequence:

We do not need to prove that the intermittent motion time $\mathrm{T}$ is the intertwining of the motion time and the immobility time of the object in time $\mathrm{T}$. We only need to prove that motion time of the object corresponds to the object moving at $C_{\max }$ in that time.

Let $\mathrm{A}$ be the object in intermittent motion at velocity $v_{A}$ in time $\mathrm{T}, v_{A}<$ $C_{\max }$

Since the motion of A is the intermittent motion in time $\mathrm{T}$, then we decompose time $\mathrm{T}$ into the sum of the immobility time $t$ and the motion time $t^{\prime}$ as follows:

$$
\begin{aligned}
& T=t+t^{\prime} \\
& v_{A-T}=\frac{S_{T}}{T}
\end{aligned}
$$

Where:

- $t^{\prime}$ is the motion time of $A$ in time $T$.

- $t$ is the immobility time of $A$ in time $T$.

- $v_{A_{-} T}$ is the velocity of $A$ in time $T$, and $v_{A_{-} T}$ represents the intermittent motion of $A$. 
- $S_{T}$ is the distance $A$ has reached in time $T$.

Because $t$ is the immobility time, so $t$ does not induce the motion of $\mathrm{A}$, and the distance A has reached is calculated only in the motion time $t^{\prime}$. Deduce the velocity $v_{A_{-} t^{\prime}}$ of A calculated by the motion time $t^{\prime}$ :

$$
v_{A_{-} t^{\prime}}=\frac{S_{T}}{t^{\prime}}>v_{A_{-} T}
$$

If $v_{A_{-} t^{\prime}}=C_{\max }$ then time $t^{\prime}$ is the motion time of $\mathrm{A}$ in time $\mathrm{T}$, deducing the consequence is proven.

If $v_{A_{-} t^{\prime}}<C_{\max }$ then $\mathrm{A}$ is in intermittent motion in time $t^{\prime}$, then contradicts with the given assumption, because now $t^{\prime}$ is still understood as the intermittent motion time of $\mathrm{A}, t^{\prime}$ is not the motion time. Therefore $t^{\prime}$ is the motion time of $\mathrm{A}$ if and only if there is no immobility time of A within time $t^{\prime}$, from this deduce the velocity of A corresponding to motion time $t^{\prime}$ is equal to $C_{\max }$.

Proving end.

From the axiom and proven consequence, we derive an important property of matter as follows:

If the matter moves at velocity $v \in\left(0, C_{\max }\right)$ in time $\mathbf{T}$, it will disappear(or not exist in matter form) when it moves at the instantaneous speed $C_{\max }$ and the matter will reappear(or exist in matter form) when it is not moving(absolute immobility).

For matter objects created through combinations of large-sized subatomic particles such as the moon, the earth, ... we cannot detect the disappearance and appearance of these this object, because subatomic particles do not disappear at the same time or appear at the same time. But the disappearance and appearance will be evident in subatomic particles such as Quarks, Electrons, ... we describe the disappearance and appearance of matter A(subatomic particle) as shown in Figure 1. 


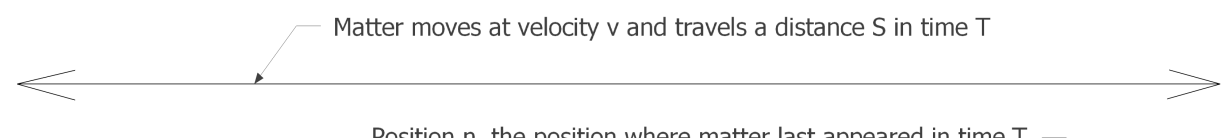

Position $n$, the position where matter last appeared in time $T$

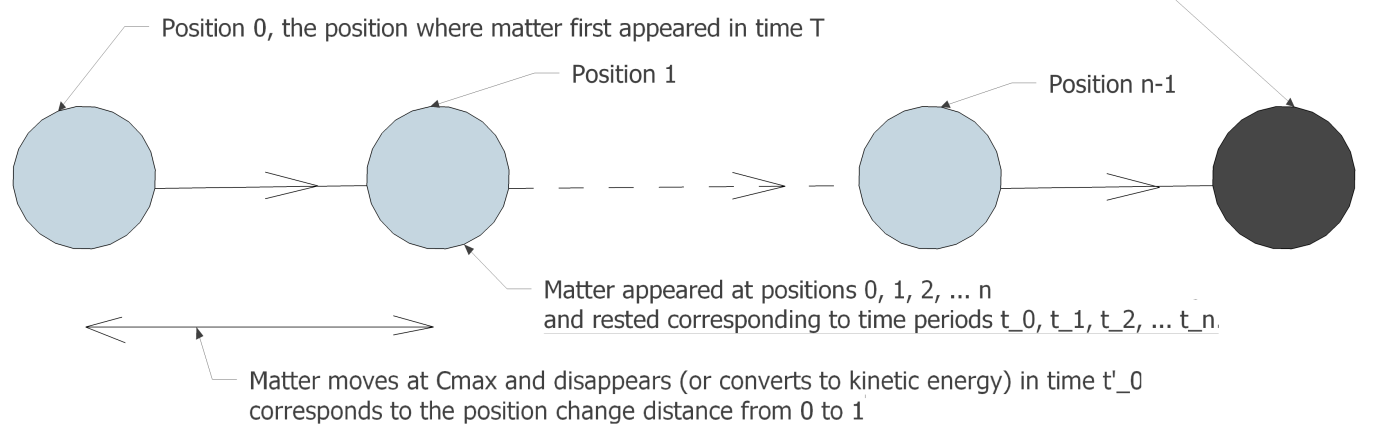

Figure 1: Describing the disappearing and appearing state of matter $A$ moving at the speed $v \in\left(0, C_{\max }\right)$ in time $T$

Where:

- When A moves at velocity v in time T, A reaches distance $S=v T$

- A will in turn change its position in space from position 0 to position $\mathrm{n}$ in time $\mathrm{T}$.

- A will appear at points corresponding to positions $0,1, \ldots$ n and A will rest(absolute immobility) at these positions on distance S. In addition, it will disappear as it moves instantaneously at $C_{\max }$.

- Let $t_{0}, t_{1}, t_{2}, \ldots t_{n-1}$ be the immobility time values of $\mathrm{A}$ when $\mathrm{A}$ appears at positions $0,1,2, \ldots n-1$. And let $t_{0}^{\prime}, t_{1}^{\prime}, t_{2}^{\prime}, \ldots t_{n-1}^{\prime}$ be the corresponding motion time values of $\mathrm{A}$ when $\mathrm{A}$ moves at $C_{\max }$.

- The intermittent motion of $\mathrm{A}$ is an alternating combination of absolute immobility in time $t_{i}, i \in N, i \in[0, n-1]$ and absolute motion at $C_{\max }$ in time $t_{i}^{\prime}, i \in N, i \in[0, n-1]$. Thus A will disappear(or not exist in matter form) in time $t_{i}^{\prime}$ because when $\mathrm{A}$ moves at $C_{\max }$ then $\mathrm{A}$ has no mass.

- Because the immobility time values $t_{i}, i \in N, i \in[0, n-1]$ do not cause the velocity of $\mathrm{A}$, so the velocity of $\mathrm{A}$ is also calculated using the expression: 


$$
\begin{gathered}
v=\frac{\sum_{i=0}^{n-1} t_{i}^{\prime}}{T} C_{\max }=\frac{S}{T} \\
T=\sum_{i=0}^{n-1}\left(t_{i}^{\prime}+t_{i}\right)
\end{gathered}
$$

Where:

- $T$ is the sum of the intermittent motion time of $A$

- $t_{i}^{\prime}$ corresponding to each discrete motion time period of $A$ in time $T, t^{\prime}=$ $\sum_{i=0}^{n-1} t_{i}^{\prime}$ is the total motion time at $C_{\max }$ of $A$ in time $T$.

- $t_{i}$ corresponding to each discrete immobility time period of $A$ in time $T$, $t=\sum_{i=0}^{n-1} t_{i}$ is the total immobility time of $A$ in time $T$.

When analyzing the intermittent motion time $\mathrm{T}$, if we remove the value $i=0$ and take the value $i=n$ then $\mathrm{T}$ can write in the form:

$$
T=\sum_{i=1}^{n}\left(t_{i}^{\prime}+t_{i}\right)=t^{\prime}+t
$$

Therefore: "If we consider mass as a measure of the existence of matter in space then matter exists only when it is motionless in space - It will disappear when it moves at $C_{\max } "$.

\subsection{The law of conservation of time}

\subsubsection{Description of time}

Time is a complex and elusive object. At present, we can only describe mind we understand about time, we have yet to build a complete concept of time. But whether the description of the time is simple or complex then the content of description must show countable ticks on the clock.

Therefore, the simplest method to describe time is by relying on countable ticks or oscillations on the clock, such as we measure time through countable vibrations of the pendulum, or one second corresponds to the 9,192 631,770 
radiation cycles of electron in the Cesium atom.

In which the nature of tick or vibration is the motion of matter, periodic oscillation of object A (matter) is understood as object A moving at constant velocity (Except for light, since light travels in vacuum independent of its frequency, we will analyze radiation formation in section 2.5.).

On the other hand, if $\mathrm{A}$ (matter) moves at a constant speed $(v=$ const $<$ $\left.C_{\max }\right)$ in time $\mathrm{T}$ then the intermittent motion time $\mathrm{T}$ exists two types of time:

- Immobility time $t$

- Motion time $t^{\prime}$

$$
T=\sum_{i=1}^{n}\left(t_{i}^{\prime}+t_{i}\right)=t^{\prime}+t
$$

In which immobility time $t_{i}$ and motion time $t_{i}^{\prime}$ are intertwined to create one cycle time $T_{\text {cycle_i }}$ :

$$
\left\{\begin{array}{c}
T_{\text {cycle_i } i}=t_{i}+t_{i}^{\prime} \\
T=\sum_{i=1}^{n} T_{\text {cycle_i }}
\end{array}\right.
$$

Because $v=$ const $<C_{\max }$, therefore:

$$
\begin{aligned}
& v=\frac{t_{i}^{\prime}}{T_{\text {cycle } \_}} C_{\max }=\frac{t^{\prime}}{T} C_{\max } \\
& \rightarrow T=\sum_{i=1}^{n} T_{\text {cycle } \_i}=n T_{\text {cycle }}
\end{aligned}
$$

Thus, in a cycle of $\mathrm{A}$, there are also two types of time:

$$
T_{\text {cycle }}=t_{\text {cycle }}+t_{\text {cycle }}^{\prime}
$$

In addition, we are based on the statement: "A appears(exists) only when A is motionless in space" - This leads to the result: If $\mathbf{A}$ is the electron in a cesium atomic clock then A will only display its immobility time value on the clock. 
Because it cannot display time when it disappears (or does not exist), or does not display time as it moves at $C_{\max }$, if we want to measure the time it disappears then we need to use another clock to measure its motion time.

This is an important argument, which is also based on Godel's incompleteness theorem to deduce the result, which is explained as follows: If you want to measure the motion time and your velocity then you need to have another object as reference, if you refer to yourself then your velocity is equal to zero so your time is your immobility time. In fact, your velocity is different from zero when you are moving, but you have no way of expressing the your motion time without taking another object as a reference.

When $\mathrm{A}$ appears and remains motionless for a time $t_{i}$ then the time $t_{i}$ will be displayed on the clock corresponding to an oscillation(or a tick). We outside will perceive the disappearance of $\mathrm{A}$ and it is represented by the expression: $T_{\text {cycle }}=t_{\text {cycle }}+t_{\text {cycle }}^{\prime}$. We know this because each of our bodies is like another clock that is watching disappearance and reappearance of object A.

This is similar to when you move at $C_{\max }$ with the clock next to it, the time displayed on the clock will be zero until you stop(or until you move at less than $C_{\max }$ ) and then the time value will be equal to one oscillate(or one tick), this oscillate represents immobility time value, not motion time value.

Suppose there is an observer stands motionless and watches you move at $C_{\max }$ for $\mathrm{T}=1$ hour, but you are an insider so you won't see you moving for an hour, the time value you get is zero, because:

$$
\begin{aligned}
& t_{\text {immobility_you }}=0 \\
& \rightarrow T=t_{\text {motion_you }}^{\prime}
\end{aligned}
$$

And the 1 hour value that the observer gets because he is standing absolutely motionless:

$$
\left\{\begin{array}{l}
t_{\text {observer }}=T \\
t_{\text {observer }}^{\prime}=0
\end{array}\right.
$$

If the observer is also moving at $C_{\max }$ at the same time you are moving at $C_{\max }$ then the observer will get your motion time with zero value: 


$$
\left\{\begin{array}{l}
t_{\text {observer }}=0 \\
t_{\text {observer }}^{\prime}=T
\end{array}\right.
$$

If the observer is also moving at $v_{\text {observer }}<C_{\max }$ at the same time you are moving at $C_{\max }$ then the observer will get your motion time:

$$
\begin{gathered}
T=t_{\text {observer }}+t_{\text {observer }}^{\prime} \\
\rightarrow \frac{t_{\text {observer }}}{T}+\frac{t_{\text {observer }}^{\prime}}{T}=1 \\
\rightarrow \frac{t_{\text {observer }}}{T}+\frac{v_{\text {observer }}}{C_{\max }}=1 \\
\rightarrow t_{\text {observer }}=T\left(1-\frac{v_{\text {observer }}}{C_{\max }}\right)
\end{gathered}
$$

1.4.2. The law of conservation of time

The content of the law of conservation of time as follows:

"The time $T$ of an object is constant when the object changes its speed and the value of $T$ is equal to the total motion time and total immobility time of the object."

$$
\begin{gathered}
T=\sum_{i=1}^{n} t_{i}+\sum_{i=1}^{n} t_{i}^{\prime} \\
\rightarrow T=t+t^{\prime}
\end{gathered}
$$

Where:

- $T$ is the total time, the time at which the object has moved at v velocity, $0 \leq v \leq C_{\max }$ 
- $t$ is the sum of immobility time corresponding to the velocity $v=0$ of the object in time $T$.

- $t^{\prime}$ is the sum of motion time corresponding to the velocity $C_{\max }$ of the object in time $T$.

The meaning of the law of conservation of time:

- Corresponding to the velocity of each object, there are always two types of immobility time and motion time for that object, but there also exists a common and equal time for all objects and does not depend on the velocity of the objects. Time $T$ is the same everywhere and exhibits uniformity and not be violated, hence the law of conservation of energy is also not violated on a cosmic scale.

- The law of conservation of time is highly applicable, it is built to solve complex problems related to time elasticity in the motion of objects, we will see its applications in the section two of the manuscript.

- Time conservation $T$ is only meaningful when we compare time $T$ between different objects corresponding to different speeds. Or when we compare two motion events of an object but at different times, then we use the common time $T$ for those two events.

The law of conservation of time has been deduced from two axioms and two consequences that the manuscript built, but the author still implements the proof method for our reference.

Prove:

Suppose two objects $\mathrm{A}$ and $\mathrm{B}$ are moving with velocity $v_{A}, v_{B}$ in time $\mathrm{T}$ $\left(0 \leq v_{A}<v_{B} \leq C_{\max }\right)$ then the velocities of $\mathrm{A}$ and $\mathrm{B}$ are calculated in time T corresponding to the expressions:

$$
\begin{gathered}
v_{A}=\frac{S_{A}}{T}=\frac{t_{A}^{\prime}}{T} C_{\max } \\
\rightarrow t_{A}=T-t_{A}^{\prime} \\
v_{B}=\frac{S_{B}}{T}=\frac{t_{B}^{\prime}}{T} C_{\max }
\end{gathered}
$$




$$
\rightarrow t_{B}=T-t_{B}^{\prime}
$$

This leads to:

$$
t_{A}-t_{B}=t_{B}^{\prime}-t_{A}^{\prime}=\Delta t=\Delta t^{\prime}
$$

Where:

- $t_{A}^{\prime}$ is the motion time of $A$ in time $T$

- $t_{B}^{\prime}$ is the motion time of $B$ in time $T$

- $\Delta t^{\prime}$ is the difference between motion time of $B$ and $A$ in time $T$

- $\Delta t$ is the difference between immobility time of $A$ and $B$ in time $T$

Thus, if $\mathrm{A}$ accelerates and moves at the speed $v_{A \_n e w}=v_{B}$ then:

$$
\begin{aligned}
& \left\{\begin{array}{l}
t_{\text {A_new }}^{\prime}=t_{A}^{\prime}+\Delta t^{\prime} \\
t_{\text {A_new }}=t_{A}-\Delta t \\
\Delta t^{\prime}=\Delta t
\end{array}\right.
\end{aligned}
$$

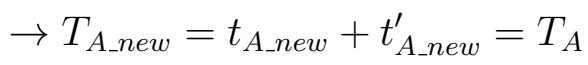

Conversely, if $\mathrm{B}$ decelerates and moves at $v_{B_{-} \text {new }}=v_{A}$ then we also have:

$$
T_{B \_n e w}=t_{B \_n e w}+t_{B \_n e w}^{\prime}=T_{B}
$$

Therefore, time $\mathrm{T}$ is constant and preserved when the object changes speed. Proving end. 


\subsection{Verify the maximum velocity in the universe.}

Maximum velocity $C_{\max }$ in the universe was proved by the author in section two with the value $C_{\max }=v_{\text {light }}=c=299,792,458 \mathrm{~m} / \mathrm{s}$. But to show independence from the speed of light, the author also proposed a new method to measure $C_{\max }$ value.

The verification method is as follows:

The first, we need to determine a distance $\mathrm{S}$ with known value, $\mathrm{S}$ is the circumference of a circle with radius $r$ at the ground.

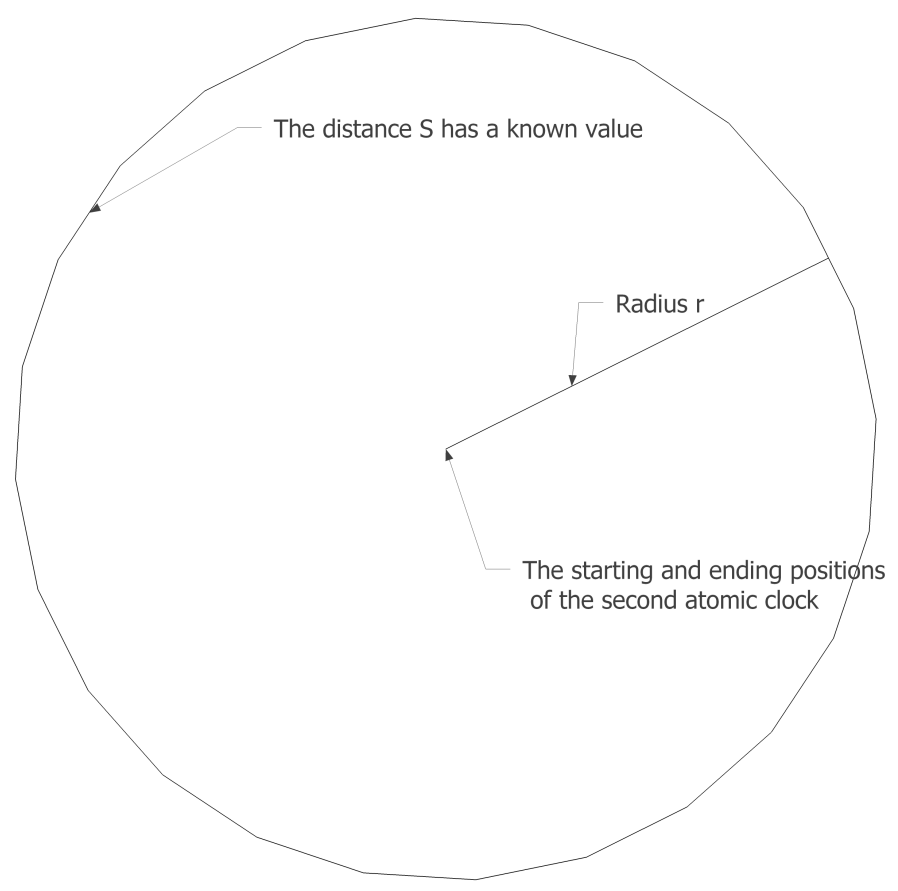

Figure 2: Describe the method to verify the maximum speed in the universe

The next, we synchronize the two atomic clocks and place at the center of the circle with the circumference $\mathrm{S}$, then an observer brings the second clock and moves all the way $S_{0}$ to return to compare time with the first clock.

The distance is located from the center of the circle, then the observer will go all the radius $\mathrm{r}$ to get to the circumference $\mathrm{S}$. Next, he goes all the way around the circle, and finally, the observer returns to the center of the circle. 
The observer's velocity is indeterminate and we are not interested in his velocity, because is calculated using the expression:

$$
\begin{gathered}
S_{0}=S+2 r \\
C_{\max }=\frac{S_{0}}{t_{1}-t_{2}}
\end{gathered}
$$

Where:

- $t_{1}$ is the time displayed on the first clock.

- $t_{2}$ is the time displayed on the second clock.

\subsection{The empirical evidences.}

1.6.1. Verification of matter disappears when moving

a. Quantum tunnelling:

"Quantum tunnelling is the quantum mechanical phenomenon where a subatomic particle passes through a potential barrier."

Explanation:

When the subatomic particle is moving at velocity $\mathrm{v}$ in time $\mathrm{T}$, time $\mathrm{T}$ of the particle is calculated using the expression:

$$
T=\sum_{i=1}^{n}\left(t_{i}^{\prime}+t_{i}\right)
$$

Time $\mathrm{T}$ is divided into cycles, which are the cycles of disappearance and appearance of the particle:

$$
\begin{gathered}
T_{i}=t_{i}+t_{i}^{\prime} \Leftrightarrow T_{\text {cycle } \_i}=t_{\text {cycle } \_}+t_{\text {cycle } i}^{\prime} \\
i \in N, i \in[1, n]
\end{gathered}
$$

If the subatomic particle moves at a constant speed then the time of the 
cycles will be the same:

$$
\begin{gathered}
T_{i}=t_{i}+t_{i}^{\prime} \Leftrightarrow T_{\text {cycle }}=t_{\text {cycle }}+t_{\text {cycle }}^{\prime} \\
\rightarrow v=\frac{t_{\text {cycle }}^{\prime}}{T_{\text {cycle }}} C_{\max }
\end{gathered}
$$

In quantum tunneling, subatomic particle are not affected by the barrier energy all the time $\mathrm{T}$, but the particle will be affected in case it gets close to the energy barrier. We consider the moment when the particle almost touches the energy barrier, then the particle will be affected by the energy barrier corresponding to one or more time cycles $T_{\text {cycle. }}$ In which, motion time $t_{c y c l e}^{\prime}$ and immobility time $t_{c y c l e}$ in time $T_{c y c l e}$ have two different functions:

- Immobility time $t_{\text {cycle }}$ functions to prevent particle motion, if $t_{\text {cycle }}$ is larger the energy barrier will act on the particle long enough, causing the particle's orbit to deviate or particle bounces back. Therefore, the necessary condition in the quantum tunnel is that the velocity of the particle must be large enough to reduce the $t_{\text {cycle }}$ value or the energy barrier has a low energy level so that the particle will not be affected for too long by the barrier.

- The motion time $t_{\text {cycle }}^{\prime}$ has the function of causing the subatomic particle to cross the energy barrier, the particle will not be prevented by the energy barrier because it moves at $C_{\max }$ in this time. Because when particle moves at $C_{\max }$ in time $t_{c y c l e}^{\prime}$, the particle disappears and reappears when the particle is not in motion. 


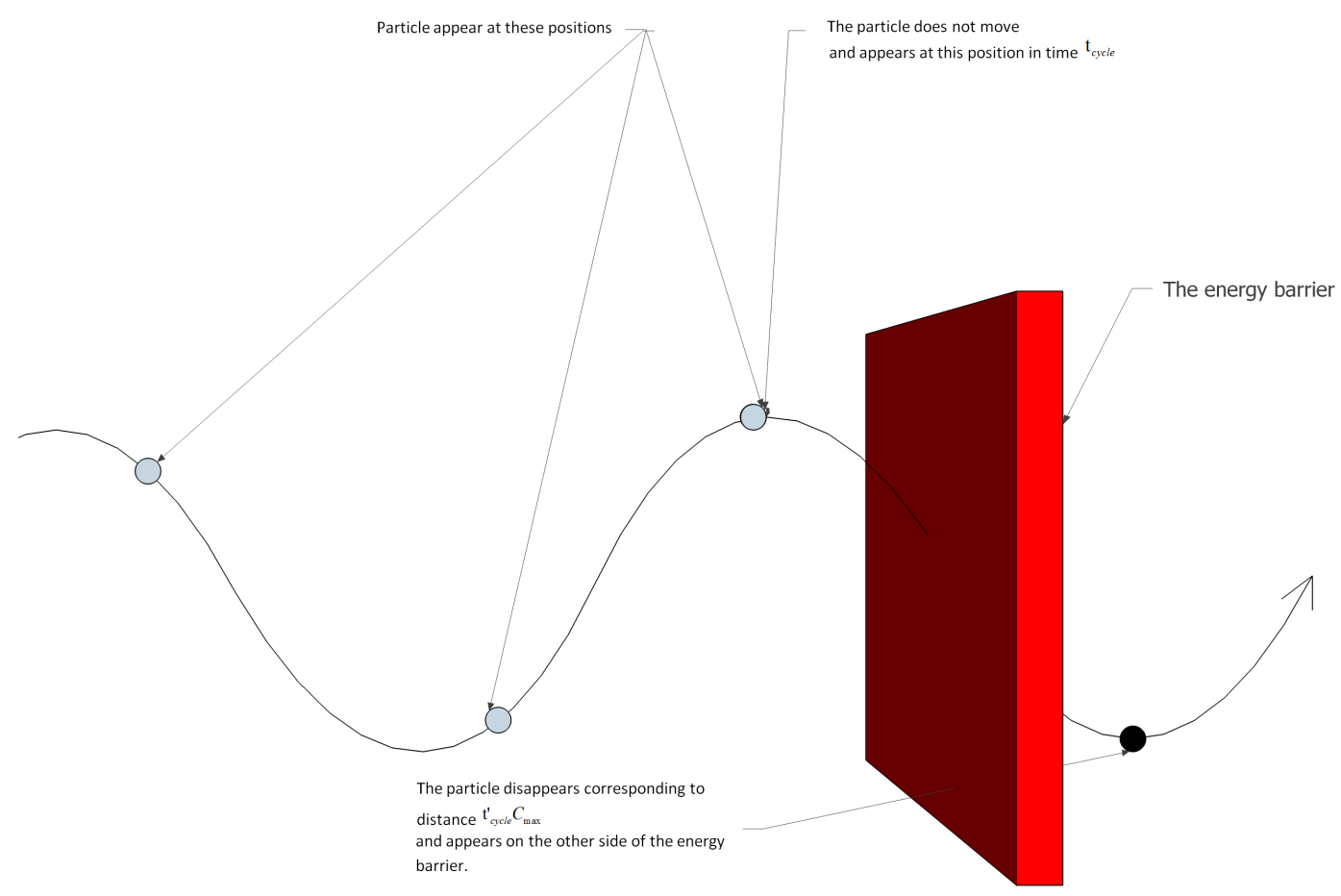

Figure 2: Describes a particle that has escaped to the other side of the energy barrier

Thus, in order for subatomic particles to cross the energy barrier, it is required that the velocity of the particle be large enough to reduce immobility time of particles. In addition, only subatomic particles can cross the energy barrier through their disappearance and appearance. For large objects formed from many subatomic particles, it will be difficult to cross the barrier of energy even though the speed of the object is very large. Because the disappearance and appearance of these objects will decrease with size, the subatomic particles will not disappear at the same time, so the object cannot disappear either.

b. Evidence of electrons disappears as they move

Scientists have discovered a strange kind of quantum motion that occurs in electrons traveling between the atomic layers of the material.

Instead of moving from the top layer to the bottom layer through the middle layer, the electrons disappear in the top layer and reappear in the lower layer, 
with no sign of electrons in the middle layer.

Reference source: Pubs.acs.org

\subsubsection{Evidence of time change is displayed on the clock as the clock changes speed}

a. The known evidences

We already have a lot of evidence of the change in time displayed on the clock when the clock changes speed, we can refer here:

Reference source: nature.com

Reference source: Wikipedia

Explanation:

Suppose if the clock displays time $\mathrm{T}$ then the time values in the clocks are equal in all motion events with different speeds, because time $\mathrm{T}$ is conserved. But the clock only shows its immobility time, so when the clock changes speed, the motion time also changes, this leads to the immobility time value displayed on the clock calculated using the expression:

$$
t_{\text {immobility }}=T_{\text {total }}-t_{\text {motion }}^{\prime}
$$

Thus, if the clock accelerates then the displayed time will have a smaller value than the non-accelerated clock.

Here, we also need to note that gravity is also a cause of the object's speed change. When we place the clock above, gravity will have less effect on the clock compared to the clock placed on the ground. We do not see the clock moving, but the subatomic particles inside the clock have changed velocity under different effects of gravity. So we say the gravity changes the time displayed on the clock, but the essence of the problem is that gravity changes the velocity of the subatomic particles inside the clocks, causing it to display different immobility time values for different clocks.

Time dilation is not really happening, the clock doesn't measure time $\mathrm{T}$ in the perfect way we want it, so if we just rely on the immobility time value shown on the clock and we conclude that if we move close to the speed of 
light then the lifespan ours will be higher than when we rest, then the author thinks that this is a hasty and controversial conclusion(because we have not realized time $T$ is conserved).

b. The real lifespan of the Muon when it falls to the earth's surface

The lifetime of Muon is about 2.2 microseconds, this value is measured according to our clock, it is the immobility time of the clock. This value does not represent the real life of the Muon, because if the Muon's lifespan is only 2.2 microseconds then it would not be able to travel $10 \mathrm{~km}$ falling from the atmosphere to the earth's surface. This absurdity is inferred because even light takes 33 microseconds to go all the way $10 \mathrm{~km}$, while the Muon particle's speed is only $0.94 \mathrm{c}$

The real life of the Muon will be calculated in time T, Muon's life as measured by the immobility time displayed on the meter is the relative value, we need to convert it back to the real value according to the conservation time $\mathrm{T}$. The solution to this problem has been presented in section 2.4.2. 


\section{The Applications of The Law of Conservation of Time}

\subsection{Construct expressions to calculate velocity and distance.}

According to the law of conservation of time, all of object in the universe has only one instantaneous velocity equal to $C_{\max }$, but the instantaneous velocity $C_{\max }$ combined with the object's immobility time creates a range of average velocities from 0 to $C_{\max }$.

The velocity of an object is only caused by time $t_{\text {motion }}^{\prime}$, so the method of calculating the average velocity of a moving object in time $\mathrm{T}$ is calculated by the expression:

$$
v=\frac{t_{\text {motion }}^{\prime}}{T_{\text {total }}} * C_{\max }
$$

Where:

- $v$ is average velocity of moving object in time $T$

- $t_{\text {motion }}^{\prime}$ is the motion time of the object with maximum speed $C_{\max }, t_{\text {motion }}^{\prime}$ depends on $T_{\text {total }}$

- $C_{\max }$ is the maximum velocity in the universe.

The above expression for velocity calculation is independent of the frame of reference(observer), because it only contains the parameters of the object itself and has no parameters related to the reference system.

This leads to an expression that calculates the distance the object has traveled in time $\mathrm{T}$ :

$$
S=t_{\text {motion }}^{\prime} * C_{\text {max }}=v T_{\text {total }}
$$

We can also rewrite the expressions in a simplified form after replacing the symbols $t_{\text {motion }}^{\prime}, t_{\text {immobility }}, T_{\text {total }}$ with the corresponding $t^{\prime}, t, T$ and:

$$
\begin{gathered}
v=\frac{t^{\prime}}{T} * C_{\max } \\
t=T\left(1-\frac{v}{C_{\max }}\right) \\
S=t^{\prime} * C_{\max }=v T
\end{gathered}
$$




\subsection{Prove that the speed of light in vacuum is the maximum speed in the universe.}

The concept of velocity(linear velocity , relative velocity) in physics today is understood as the speed of changing the position of the object with respect to the frame of reference. For each frame of reference, the obtained velocity value may differ depending on the motion velocity of the frame of reference(observer).

Thus the velocity of the object that the concept is referring to is essentially the difference of distance by immobility time of the reference system, so it is relativistic. The relative velocity is different from the real velocity of the object, and the relative velocity is calculated using the expression:

$$
v_{\text {relativity_object }}=\frac{t_{\text {object }}^{\prime}-t_{\text {observer }}^{\prime}}{T-t_{\text {observer }}^{\prime}} * C_{\text {max }}
$$

Where:

- $v_{\text {relativity_object }}$ is the relative velocity of the object calculated by the observer's measurement.

- $t_{\text {object }}^{\prime}$ is the motion time of the object's $C_{\max }$ speed in time $T$.

- $t_{\text {observer }}^{\prime}$ is the motion time of the observer's $C_{\max }$ speed in time $T$.

- T is the total time, time for an observer to take the measurement.

- $t_{\text {observer }}=T-t_{\text {observer }}^{\prime}$ is the time value displayed on the observer's clock.

From the expression for the relative velocity, we see the value of the relative velocity is smaller than the real velocity of the object: $v=\frac{t_{\text {object }}^{\prime}}{T_{\text {total }}} * C_{\text {max }}$. And the value of the relative velocity will be equal to the real velocity if and only if the object moves at $C_{\max }$, this can be stated and substantiated as follows:

Statement:

"If an object moves at $C_{\max }$ then the relative velocity is constant, independent of the observer's speed - Otherwise, if the relative velocity of the object has the value is constant, independent of the observer's speed, then the object is moving at $C_{\max }$ " 


\section{$\underline{\text { Prove: }}$}

Prove the first side: if the object moves at $C_{\max }$ then the relative velocity is constant for all observers.

The difference in distance between observer and object in time $\mathrm{T}$ :

$$
\Delta s=S_{\text {object }}-S_{\text {observer }}
$$

Where:

- $S_{\text {object }}$ is the distance reached by the object in time $T$, it is calculated by the expression: $S_{\text {object }}=t_{\text {object }}^{\prime} * C_{\mathrm{max}}$

- Sobserver is the distance reached by observer in time $T$, it is calculated by the expression: $S_{\text {observer }}=t_{\text {observer }}^{\prime} * C_{\max }$

Since the object moves at $C_{\max }$ in time $\mathrm{T}$, so all of time $\mathrm{T}$ of the object have been converted to $t_{\text {object }}^{\prime}$ :

$$
t_{\text {object }}^{\prime}=T
$$

Lead to:

$$
\begin{gathered}
\Delta s=T * C_{\max }-t_{\text {observer }}^{\prime} * C_{\max }=t_{\text {observer }} * C_{\max } \\
\rightarrow v_{\text {relativity_object }}=\frac{\Delta s}{T-t_{\text {observer }}^{\prime}}=\frac{t_{\text {observer }} * C_{\max }}{t_{\text {observer }}}=C_{\max }
\end{gathered}
$$

Conclusion: The relative velocity value is obtained by constant $v_{\text {relativity_object }}=$ $C_{\text {max }}$, which is independent of all observers' velocities.

Prove the second side: If the relative velocity of the object is constant for all observers then the object is moving at speed $C_{\max }$.

Because the object 's relative velocity is independent of the observer's speed, therefore:

$$
v_{\text {relativity_object }}=\frac{t_{\text {object }}^{\prime}-t_{\text {observer }}^{\prime}}{T-t_{\text {observer }}^{\prime}} * C_{\text {max }}=\frac{t_{\text {object }}^{\prime}-\left(t_{\text {observer }}^{\prime}+\Delta t^{\prime}\right)}{T-\left(t_{\text {observer }}^{\prime}+\Delta t^{\prime}\right)} * C_{\text {max }}
$$

Where $\Delta t^{\prime} \neq 0$ represents the observer's different speeds in the object velocity measurements: 


$$
\frac{t_{\text {observer }}^{\prime}}{T} C_{\max } \neq \frac{t_{\text {observer }}^{\prime}+\Delta t^{\prime}}{T} C_{\max }
$$

Solving equation 1, we get the result: $t_{\text {object }}^{\prime}=T$

This results in the speed of the object receiving the value:

$$
v=\frac{t_{\text {object }}^{\prime}}{T} C_{\max }=\frac{T}{T} C_{\max }=C_{\max }
$$

Proving end.

Replacing the object with light and the speed of light in vacuum, we draw the following conclusion: The relative speed of light in vacuum has been verified to be independent of all observers' speeds, so the speed of light in vacuum has a value $v_{\text {light }}=C_{\max }=299,792,458 \mathrm{~m} / \mathrm{s}$.

The speed of light in vacuum reaches $C_{\max }$ but we still see light, which can be explained that the light is motionless the moment it hits our eyes (or touches the sensors of the measuring equipment). Sensors of the measuring devices only record the result value at the present time, not record the results in the past or future, hence our eyes(or sensors) cannot perceive light until it touches and motionless for a moment. This has also been verified experimentally when our observations affect the wave properties of a particle or light, the particle's wave properties will be significantly reduced(less interference, wave properties decrease while particle properties increase) if one or more detectors are observing the particle's motion.

From the expression for the relative velocity, we can derive the relationship between the relative velocity and the real velocity through the expression:

$$
\begin{gathered}
v_{\text {relativity_object }}=\frac{t_{\text {object }}^{\prime}-t_{\text {observer }}^{\prime}}{T-t_{\text {observer }}^{\prime}} * C_{\max } \\
\rightarrow v_{\text {real_object }}=v_{\text {observer }}+\left(1-\frac{v_{\text {observer }}}{C_{\max }}\right) * v_{\text {relativity_object }}
\end{gathered}
$$

Equation 2 shows the relationship between the relative velocity and the real velocity of the object, the $v_{\text {relativity_object }}$ is measured by an observer moving at real velocity $v_{\text {observer }}$ 
$\underline{\text { Note: }}$

Relative velocity is not the real velocity of the object, the relative velocity is also not the value of the difference between real velocity of the object and real velocity of the frame of reference.

Because the relative velocity is calculated by the expression:

$$
v_{\text {relativity_object }}=\frac{\Delta s}{t_{\text {observer }}}
$$

While the velocity difference value of two objects is calculated by the expression:

$$
\Delta v=\frac{\Delta s}{T_{\text {total }}}
$$

Hence the relative velocity is always greater than $\Delta v$ and equal to:

$$
\Delta v=\frac{t_{\text {observer }}}{T_{\text {total }}} * v_{\text {relativity_object }}=\left(1-\frac{v_{\text {observer }}}{C_{\max }}\right) * v_{\text {relativity_object }}
$$

\subsection{The distance measurement results are also relative.}

We refer to the definition of meters in physics: "The metre is defined as the length of the path travelled by light in a vacuum in 1/299,792,458 of a second". Where the meter is the base unit for measuring length or distance.

Hence distance is a concept that takes the speed of light as a standard and it is calculated by the expression:

$$
S=t_{\text {observer }} C_{\max }
$$

Where:

- $S$ is the distance(or length) to be measured

- $t$ is the time value for which light has traveled the distance $S$, time is based on our clock - immobility time. 
- $C_{\max }$ is the speed of light in vacuum, the maximum velocity in the universe.

This results in the distance measurement being relative, because it depends on the observer's immobility time(frame of reference).

To understand why distance values are relativistic and dependent on a frame of reference, we need to reconsider the expression for the relative velocity of light:

$$
\begin{aligned}
& v_{\text {light }}=\frac{t_{\text {light }}^{\prime} * C_{\mathrm{max}}-t_{\text {observer }}^{\prime} * C_{\mathrm{max}}}{T-t_{\text {observer }}^{\prime}} \\
& \rightarrow v_{\text {light }}=\frac{T * C_{\mathrm{max}}-t_{\text {observer }}^{\prime} * C_{\mathrm{max}}}{t_{\text {observer }}}
\end{aligned}
$$

In equation 3 , the relative speed of light is always constant for all observers $\left(v_{\text {light }}=\right.$ $C_{\max }$ ), while the light's travel distance value depends on the speed of each observer:

$$
S_{\text {light }}=v_{\text {light }} * t_{\mathrm{observer}}=T * C_{\mathrm{max}}-t_{\mathrm{observer}}^{\prime} * C_{\mathrm{max}}=C_{\mathrm{max}} * t_{\mathrm{observer}}
$$

And value $C_{\max } * t_{\text {observer }}$ has been used as a standard measure to calculate distance(or length), so the value of distance is relative, depending on the motion velocity of the frame of reference.

If we call this the "Length contraction" then length contraction is independent of the observer's direction of motion, this length contraction takes place in all directions of the observer's motion. Because any observer's immobility time in time $\mathrm{T}$ depends only on the observer's speed and not on the observer's direction of motion.

We explain this effect with an example like this:

We measure the length $\mathrm{L}$ of a stadium in terms of the speed of light (for ease of calculation, we take $C_{\max }=3 * 10^{8} \mathrm{~m} / \mathrm{s}$ ). Assume that the earth in this case is at absolute rest and we get $t_{\text {earth_1 }}=5 * 10^{-7}$ second for light to travel to its length. Hence the length $\mathrm{L}$ of the stadium in this case has a value of $\mathrm{L}=150 \mathrm{~m}$.

Assume the future earth has a speed of $v_{\text {earth }}=0.9999 C_{\max }$. If we measure the length $\mathrm{L}$ of the stadium in this case then the stadium length is only equal 
to $\mathrm{L}=0.15 \mathrm{~m}$.

The causes of the "length contraction" effect are explained as follows:

- In both cases the light travels for the full length of the stadium with an equal T time value: $T=5 * 10^{-7}$ second.

- But in the first case the immobility time displayed on the observer's clock is equal to $T$ because the earth is absolutely stationary, therefore:

$$
L=t_{\text {earth } \_1} C_{\max }=T C_{\max }=5 * 10^{-7} * 3 * 10^{8}=150
$$

- Moving to the case where the earth speed is equal to $v_{\text {earth }}=0.9999 C_{\max }$, at this time light also completes distance $L$ with time $T$, but the observer's immobility time only records the following value:

$$
\begin{aligned}
& \frac{t_{\text {earth_2 }}}{T}=1-\frac{v_{\text {earth }}}{C_{\max }}=1-\frac{0.9999 C_{\max }}{C_{\max }}=10^{-3} \\
& \rightarrow t_{\text {earth } \_2}=10^{-3} * 5 * 10^{-7}=5 * 10^{-10} \\
& \rightarrow L=t_{\text {earth } \_2} C_{\max }=5 * 10^{-10} * 3 * 10^{8}=0.15
\end{aligned}
$$

To overcome the time change caused by motion, we have built a good solution that is to assign the standard time unit(1 second) corresponding to $9,192,631,770$ radiation cycles of the caesium- 133 atom $\left({ }^{133} \mathrm{Cs}\right)$. This means that if we synchronize the time according to the ${ }^{133} \mathrm{Cs}$ standard before measuring the stadium length in second case then after measuring we get the results of the time, and the length $\mathrm{L}$ still has the value $\mathrm{L}=150 \mathrm{~m}$ in second case.

But the time synchronization according to ${ }^{133} \mathrm{Cs}$ standard before taking the measurement means that we have ignored the change in speed of the earth in the second case. In addition, the nature of immobility time remains unchanged even if we perform synchronization, this means that after the time is synchronized, we still call the time unit(second) in second case, but in reality this time unit is larger when compared to the time unit in first case(The second unit is now only the same form but different in essence.)

Further, any observer co-moving with the observed object cannot measure the object's contraction. Because everything around observer is also affected 
by the effect of "length contraction" , the effect of "length contraction" is only observed when there is a comparison between the rest frame and the moving frame or frames with different velocities.

\subsection{Solve the problem of Muons falling on the surface of the earth.}

2.4.1. Distinguish two types of immobility time in one clock.

Maybe some people think that: "The immobility time of the frame of reference system is the time displayed on the clock of the frame of reference". But this needs careful analysis, because if we assume that a frame of reference is corresponding to an observer then the observer's immobility time is not the time displayed on his clock.

We already know that the immobility time of the object is closely related to the speed of that object according to the expression:

$$
t_{\text {immobility }}=T_{\text {total }}\left(1-\frac{v}{C_{\max }}\right)
$$

But if we analyze the velocity of an object then two kinds of velocities appear in the object: The velocity of the particles that make up the object and the velocity of the object's shell, such as velocity of the earth is different from the velocity of the subatomic particles constituting the earth,... the velocity of the watch case differs from the velocity of the matter particles that make the clock.

Therefore if we analyze the time displayed on the atomic clock ${ }^{133} \mathrm{Cs}$ then that time will correspond to the velocity of the oscillating electron with radiation frequency 9,192,631,770. Because we need to understand that when the earth(or clock) is in motion it does not exhibit the property of disappearing - appearing, this property only in subatomic particles. Therefore the objects themselves (earth, clock, ...) will not be able to express their own time, the velocity of object will depend on the velocity of the particles.

This relationship of internal and external velocity is that there is no theory or equation describing them yet. But we still mention to avoid misunderstanding when solving problems related to the immobility time displayed on the clock, the velocity corresponding to that immobility time is not the velocity 
of the observer.

In doing this analysis, we also discover a small flaw when synchronizing time according to the standards of the atomic clock Cesium: "We have unintentionally synchronized the particle velocity of all clocks, therefore the particle velocity of all clocks will have the same value". For example, the radiation frequency of ${ }^{133} \mathrm{Cs}$ is smaller than the radiation frequency of Ytterbium ion, so the velocities of the particles in these two clocks are different but they both exhibit the same time values - This leads to a contradiction even if we use the theory of relativity to evaluate.

Because the time nature is changed when we do time synchronization of the clocks. So if we concept that one second displayed on ${ }^{133} \mathrm{Cs}$ clock corresponds to the immobility time of the electron in ${ }^{133} \mathrm{Cs}$ then the time of one second on other clocks does not reflect the velocity or immobility time of the particles in those clocks(Ytterbium ion clock, calcium ion clock, strontium mesh clock,...).

Finally, to verify that the time displayed on the ${ }^{133} \mathrm{Cs}$ clock is that of the electron $v_{e}$, we will perform the measurement as follows: There are two identical atomic ${ }^{133} \mathrm{Cs}$ clocks and electrons having the same standard radiation period, we use the second clock to measure the relative velocity of the electron in the first clock. If the result returns the value $v_{\text {relativity } \_}=C_{\text {max }}$ (or $\left.v_{\text {relativity_e }}=0\right)$ then the time displayed on the clock is the immobility time of the electron, because the relative velocity of the electron is calculated by the expression:

$$
\begin{gathered}
v_{\text {real_object }}=v_{\text {observer }}+\left(1-\frac{v_{\text {observer }}}{C_{\max }}\right) * v_{\text {relativity_object }} \\
\rightarrow v_{e}=v_{\mathrm{e}}+\left(1-\frac{v_{\mathrm{e}}}{C_{\max }}\right) * v_{\text {relativity_e }} \\
\rightarrow v_{e}=C_{\max } \\
\rightarrow v_{\text {relativity_e }}=C_{\max }
\end{gathered}
$$

Conversely, if the result returns $0<v_{\text {relativity_e }}<C_{\max }$ then the time displayed on the clock is the immobility time of the clock case, and also the immobility time of the observer. 
$\underline{\text { Note: }}$

- The value $v_{\text {relativity_e }}=C_{\max }\left(\right.$ or $\left.v_{\text {relativity_e }}=0\right)$ in this case is the result of a self-referencing paradox in which we used the immobility time of the electron to make its velocity measurement. This result is similar if you were measuring your relative velocity based on your frame of reference then $v_{\text {relativity_e }}=0$.

- We illustrate this paradox with the story of a Pinocchio boy and long nose, every time he lies then his nose grows longer, what will happen after that if Pinocchio says: "My nose grows now"? The answer would be a paradox if you answered "it grows" or answered "it does not grow". - Reference source: Wikipedia.

- Therefore we do not care the values $v_{\text {relativity_e }}=C_{\max }\left(\right.$ or $\left.v_{\text {relativity_e }}=0\right)$ is true or false, what we need is that if the experimental result is the same as in theory then the time displayed on the clock is the immobility time of the electron.

2.4.2. Applying equations to solve the problem of Muons falling to the surface of the earth.

Hypothesis of the Muon particle problem:

Muon particles have an average lifespan of $t_{e}=2.2 * 10^{-6} \mathrm{~s}$, Muon life is calculated based on atomic clock ${ }^{133} C s$ with real electron velocity $v_{e}$. The relative velocity of Muon $v_{\text {relativity_Muon }}=0.94 C_{\max }$, and it moves from the atmospheric peak to the surface of the earth: $S=10^{4} \mathrm{~m}$.

The Muon particle problem has caused controversy for a long time in science, because even if the $\operatorname{light}\left(v_{\text {light }}=C_{\max }\right)$ goes all the way $10 \mathrm{~km}$ then the light will take $t=33 * 10^{-6} \mathrm{~s}$, while the Muon particle life is $t=2.2 * 10^{-6} \mathrm{~s}$.

Solve the problem:

The distance the Muon particle has traveled is calculated by the expression:

$$
S=v_{\text {muon }} T
$$

Where: 
- $S$ is the distance that the Muon particle has reached, $S=10^{4} \mathrm{~m}$.

- $v_{\text {muon }}$ is the real velocity of the Muon.

- $T$ is the real lifespan of the Muon.

We should note that $t_{e}=2.2 * 10^{-6}$ is not the real lifespan of the Muon, the real life of the Muon particle is determined by the time $\mathrm{T}$, not the time displayed on the ${ }^{133} \mathrm{Cs}$ clock. T is time independent of any frame of reference, where $\mathrm{T}$ is determined through a calculated $t_{e}=2.2 * 10^{-6} \mathrm{~S}$ life on the ${ }^{133} \mathrm{Cs}$ clock.

$$
v_{\text {muon }}=\frac{S}{T}=\frac{S}{t_{e}}\left(1-\frac{v_{e}}{C_{\max }}\right)
$$

Where:

- $t_{e}$ is the life of the Muon calculated according to the immobility time displayed on the clock.

- $v_{e}$ is the real velocity of the electron inside the clock.

On the other hand, we have the relationship between the relative velocity and the real velocity of the Muon particle as follows:

$$
v_{\text {Muon }}=v_{\mathrm{e}}+\left(1-\frac{v_{\mathrm{e}}}{C_{\max }}\right) * v_{\text {relativity_Muon }}
$$

Substituting the values $v_{\text {muon }}, C_{\max }, v_{\text {relativity_Muon }}$ into equation 4 , we derive the real velocity of the electron:

$$
\begin{aligned}
\frac{S}{t_{e}}\left(1-\frac{v_{e}}{C_{\max }}\right) & =v_{\mathrm{e}}+\left(1-\frac{v_{\mathrm{e}}}{C_{\max }}\right) * v_{\text {relativity_Muon }} \\
\rightarrow v_{\mathrm{e}}= & \frac{\frac{S}{t_{e}}-v_{\text {relativity_Muon }}}{1+\frac{S}{t_{e} C_{\max }}-\frac{v_{\text {relativity_Muon }}}{C_{\max }}} \\
& \rightarrow v_{\mathrm{e}} \approx 2.8 * 10^{8}
\end{aligned}
$$

Where: 
$-S=10^{4} \mathrm{~m}$

$-t_{e}=2.2 * 10^{-6} \mathrm{~s}$

- $v_{\text {relativity_Muon }}=0.94 C_{\max } \mathrm{m} / \mathrm{s}$

- $C_{\max }=3 * 10^{8} \mathrm{~m} / \mathrm{s}$

We can derive the real lifespan and real velocity of the Muon particle after having the real velocity value of the electron:

$$
\begin{gathered}
\frac{t_{e}}{T}=\left(1-\frac{v_{e}}{C_{\max }}\right) \\
\rightarrow T=t_{e}\left(1-\frac{v_{e}}{C_{\max }}\right)^{-1} \approx 33.48 * 10^{-6} \\
\rightarrow v_{\text {muon }}=\frac{S}{T} \approx 2.986 * 10^{8}
\end{gathered}
$$

Conclude:

The real lifespan of the Muon particle is $T \approx 33.48 * 10^{-6}(\mathrm{~s})$ and the Muon moves at real velocity $v_{\text {muon }} \approx 2.986 * 10^{8}(\mathrm{~m} / \mathrm{s})$ in time $\mathrm{T}$ to travel the distance from the top of the atmosphere to the surface of the earth $S=10 \mathrm{~km}$.

\subsection{Explore the red shift effect but without the increase in dis- tance between objects.}

In current physics, the red shift is an effect that causes an increase in wavelength or a decrease in frequency when the moving object moves away from the observer. The effect demonstrates an increase in the distance between the objects while the velocity of the objects is constant.

In this section, we go deeper into the red shift(or blue shift). The effect occurs while the distance of the objects is constant, but the velocity of the objects changes.

Circumstances where one of the two objects changes velocity while the distance between them does not change can be mentioned as the object moving around another object with a circular orbit, or an object rotates itself around the axis in which the angular velocity varies. 
Such motion patterns, in which the distance between objects is almost unchanged. We will build two cases with initial assumptions and deduce the results from the given assumptions.

Case 1:

An observer has a variable velocity from $v_{\text {observer } \_1}$ to $v_{\text {observer } 2}$ and is moving around the radiation $\operatorname{source}\left(v_{\text {source }}=\right.$ const $)$ in a uniform circular orbit. As a result, the observer will receive a red shift (or blue shift) in proportion to his change in velocity.

Prove:

Because the velocity of the source is constant, and if the distance from the observer to the source is constant then the total number of light pulses(or radiation frequency) received by the observer will be equal over the same T-intervals.

Expression for calculating radiation frequency:

$$
f_{0}=\frac{n}{T_{\text {total }}}
$$

Where:

- $f_{0}$ is the radiation frequency, $f_{0}$ is calculated by time $T_{\text {total }}$.

- $n$ is the total number of pulses of radiation in time $T_{\text {total }}$.

The measurement of the radiation frequency by time $T_{\text {total }}$ is only performed in theory, because the $T_{\text {total }}$ is not displayed on the observer's clock, instead his immobility time t corresponds to $T_{\text {total }}$.

For the same $T_{\text {total }}$ time value in all cases, but if the observer's velocity is in each case different then the immobility time displayed on the clock will not be the same. This has caused an effect of varying the radiation frequency when the observer makes a measurement in cases where the observer's velocity changes.

For example:

Suppose someone throws a ball up high and when the ball falls down he throws it up again, his pitching speed is constant. This event is an objective event for all observers, and assuming that he makes 100 pitches with $T_{\text {total }}=10$ seconds, we call $T_{\text {total }}$ as objective time, $T_{\text {total }}$ is the same for all observers. 
Alice was beside the pitcher and began to take measurements. But since Alice is moving around the pitcher at $v_{\text {Alice }}=0.5 C_{\max }$, so when $T_{\text {total }}$ time passes to the 10th second, Alice only received 5 seconds. This means that Alice took the measurement and obtained the following results: "He makes 100 pitches in 5 seconds, pitching frequency was 20 times/second".

Bob is a friend of Alice and is also taking the measurement. But since Bob is moving around the pitcher at $v_{B o b}=0.9999 C_{\max }$, so when the ball is thrown for the 100th then Bob's measured immobility time is $t_{B o b}=0.0001 \mathrm{~s}$. Finally, Bob writes an email to Alice and concludes: "He made 100 pitches in just 0.0001 second - pitching frequency was 1,000,000 times/second".

Consequently, the observer will receive $\mathrm{n}$ pulses of light in immobility time $\mathrm{t}$, and the observer will measure the radiation frequency based on his immobility time:

$$
f_{\text {light_observer }}=\frac{n}{t_{\text {observer }}}
$$

We have the radiation frequency difference based on the ratio:

$$
\begin{gathered}
\frac{f_{\text {light_observer }}}{f_{0}}=\frac{T}{t_{\text {observer }}}=\frac{1}{\left(1-\frac{v_{\text {observer }}}{C_{\text {max }}}\right)} \\
\rightarrow f_{\text {light_observer }}=f_{0}\left(1-\frac{v_{\text {observer }}}{C_{\max }}\right)^{-1}
\end{gathered}
$$

Apply the expression to calculate the radiation frequencies $f_{1}, f_{2}$ corresponding to the velocity $v_{\text {observer_1 }}$ and $v_{\text {observer_ } 2}$ :

$$
\begin{aligned}
& f_{1}=f_{0}\left(1-\frac{v_{\text {observer_1 }}}{C_{\max }}\right)^{-1} \\
& f_{2}=f_{0}\left(1-\frac{v_{\text {observer_-2 }}}{C_{\max }}\right)^{-1} \\
& \rightarrow \frac{f_{2}}{f_{1}}=\frac{C_{\max }-v_{\text {observer_1 }}}{C_{\max }-v_{\text {observer_2 }}}
\end{aligned}
$$

Conclusion: If the observer moves with positive acceleration $\left(v_{\text {observer_2 }}>\right.$ $\left.v_{\text {observer_1 } 1}\right)$ then he get the blue shift effect; or observer will get red shift 
effect if he moves with negative acceleration $\left(v_{\text {observer_2 }}<v_{\text {observer_1 }}\right)$

Proving end.

Case 2:

"A source of radiation moves around the observer $\left(v_{\text {observer }}=\right.$ const $)$ in a uniform circular orbit. If the source of the radiation changes velocity from $v_{\text {source_1 }}$ to $v_{\text {source_2 }}$ then the observer will receive a red shift effect(or blue shift)".

In physics, the radiation is formed due to variations in the electromagnetic fields of the particles and the wave properties of radiation are the basic property of radiation.

But if based on the explanation of the nature of electromagnetic radiation as above then it would be difficult to explain the red shift effect in this case. Therefore, the author applies the law of conservation of time to explain the formation and wave nature of radiation according to a new approach and solved the problem. This explanation does not contradict the method of explaining the nature of radiation in physics.

\section{a. Explain the formation of radiation:}

When the particle moves, it disappears and reappears. The process of disappearance and the appearance of particle is intertwined to form a cycle of disappearance and the appearance of particle.

If the matter particle moves at a constant speed, the time $\mathrm{T}$ of the particle is calculated by the expression:

$$
T=n * T_{\text {cycle }}
$$

Where:

- $T_{\text {cycle }}$ is the cycle of disappearance and the appearance of particle.

- $n$ is the total number of cycles of disappearance and the appearance of the particle in time $T$.

Inside time $T_{\text {cycle }}$, we also have: 


$$
T_{\text {cycle }}=t_{\text {cycle }}+t_{\text {cycle }}^{\prime}
$$

Where:

- $t_{\text {cycle }}$ is the appearance time of the particle in time $T_{\text {cycle }}$

- $t_{\text {cycle }}^{\prime}$ is the disappearance time of the particle in time $T_{\text {cycle }}$

The velocity of the particle is also calculated using the expression:

$$
v=\frac{t_{\text {cycle }}^{\prime}}{T_{\text {cycle }}} C_{\max }=\frac{t^{\prime}}{T} C_{\max }
$$

The frequency of particle appearance is calculated by the expression:

$$
f=\frac{1}{T_{\text {cycle }}}=\frac{n}{T}
$$

When the matter particle appears in space, it takes up space and produces a vacuum oscillation, the vacuum oscillation propagates in all directions. Therefore, the frequency of the vacuum oscillation is also the frequency of the particle's occurrence.

Analyzing this vacuum oscillation, we find it to be radiant of the particle, because:

- The vacuum oscillation has no mass.

- The vacuum oscillation has momentum and energy, its energy is calculated according to the frequency of the particle.

- The velocity of vacuum oscillation is equal to $C_{\max }$.

- The vacuum oscillation is not the source.

- The vacuum oscillator carries the particle's information.

Therefore, it can be concluded that vacuum oscillators are the radiation of particle, and the method to explain the radiation origin of the particle as above did not contradict the current explanation.

Thus, a pulse of radiation is a vacuum oscillation and the frequency of the radiation is the frequency of the particle's occurrence.

The wavelength of radiation is calculated by the expression: 


$$
\begin{gathered}
\lambda=T_{\text {cycle }} C_{\max } \\
\rightarrow v_{\text {radiation }}=\lambda f=T_{\text {cycle }} C_{\max } \frac{1}{T_{\text {cycle }}}=C_{\max }
\end{gathered}
$$

The wavelength value of radiation differs from the distance the matter particle has reached. Wavelength is measured in $T_{\text {cycle }}$ but the particle's distance is only $t_{\text {cycle }}^{\prime}$ :

$$
\begin{aligned}
& s_{\text {cycle }}=t_{\text {cycle }}^{\prime} * C_{\max } \\
& \rightarrow v_{\text {particle }}=f s_{\text {cycle }}
\end{aligned}
$$

$s_{\text {cycle }}$ is a kind of particle's wavelength, but unlike other wavelengths, connecting all adjacent appearing positions of the particle for a while $\mathrm{T}$ then it creates a curve and we call that curve the appearance orbit of the particle.

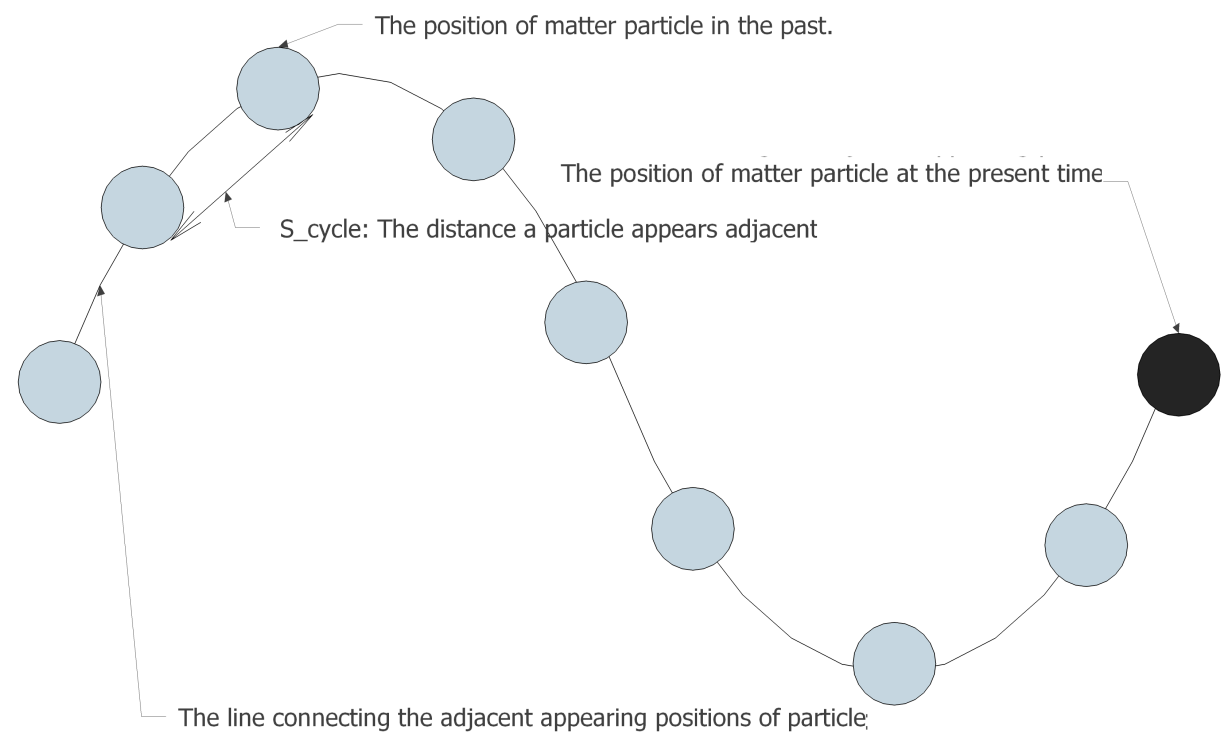

Figure 3: Describe appearance orbit of the particle

b. Explain the red shift effect when the source of radiation changes its velocity.

Thus the velocity of the particle is also proportional to the radiation frequency of the particle $v_{\text {particle }}=f_{\text {radiation }} s_{\text {cycle }}$. Set the default radiated 
frequency $f_{0}=1(\mathrm{~Hz})$ when the radiation source is absolute immobility, then the radiation frequency will be proportional to the velocity of the source according to the expression:

$$
\begin{gathered}
f=f_{0} \frac{1}{\left(1-\frac{v_{\text {source }}}{C_{\max }}\right)} \\
\rightarrow T_{\text {cycle }}=\frac{C_{\max }-v_{\text {source }}}{C_{\max } f_{0}}
\end{gathered}
$$

The observer's velocity is constant, and the distance between the two objects does not change. Let $f_{1}, f_{2}$ be the radiation frequency measured by the observer according to the velocity $v_{\text {source_1 }}$ and $v_{\text {source_2 }}$ of the radiation source, we have:

$$
\begin{aligned}
& f_{1}=\frac{1}{t_{\text {cycle_1 }}}=\frac{1}{T_{\text {cycle_1 }}\left(1-\frac{v_{\text {observer }}}{C_{\max }}\right)} \\
& f_{2}=\frac{1}{t_{\text {cycle_2 }}}=\frac{1}{T_{\text {cycle_2 }}\left(1-\frac{v_{\text {observer }}}{C_{\max }}\right)} \\
& \rightarrow \frac{f_{2}}{f_{1}}=\frac{T_{\text {cycle_1 }}}{T_{\text {cycle_2 }}}=\frac{C_{\max }-v_{\text {source_1 }}}{C_{\max }-v_{\text {source_2 }}}
\end{aligned}
$$

Conclusion: If the source moves with positive acceleration $\left(v_{\text {source_2 }}>v_{\text {source_1 }}\right)$ then the observer get the blue shift effect; or observer will get red shift effect if the source moves with negative acceleration $\left(v_{\text {source_2 }}<v_{\text {source_1 } 1}\right)$.

\section{Comment on case 2:}

- We test the red shift effect ie we examine the change in the frequency of light(radiation), not the frequency change of the radiation source. Therefore, if you explain the nature of the radiation according to the current physics then it will not find the relationship between the radiation frequency and the frequency of the source.

- The theory of radiation formation also explains why the speed of light in vacuum is independent of its wavelength, whereas the velocities of matter objects depend on their frequency or wavelength.

- It is difficult for us to measure the size of a subatomic particle, because we 
do not know its exact position, its position and velocity follow the Heisenberg uncertainty principle(the principle of experimental science). But we can measure the size of subatomic particles through the indirect method: "Make measurements based on pulse amplitude of radiation", the size of an atomic particle is very small, so it can be considered as a sphere, then the subatomic particle's radius is equal to the amplitude of the radiation pulse it emits. This can also be seen as a proof of the validity of the "formation of radiation" theory, which shows that two subatomic particles of different masses will result in different radiation amplitudes(in the absence of interference or resonance).

\subsection{Michelson-Morley experiment explanation}

\subsubsection{Description of the experiment}

The experiment was carried out in 1987 by professors Michelson and Morley, the original purpose of the experiment was to confirm the existence of Ether in space, but the experimental results created a problem in physics.

The results of the experiment showed that: "Light propagates in two different directions with different distances but with the same results of time".

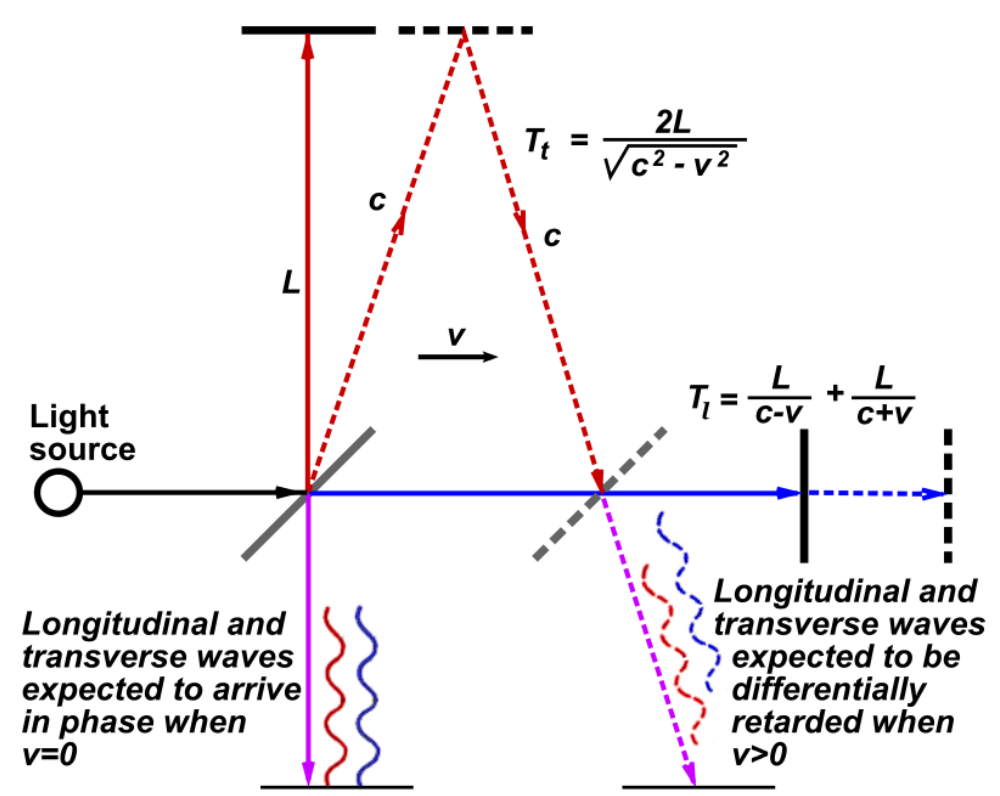

Figure 4: Michelson-Morley experiment-source: Wikipedia 
In which, the distance for the light to travel vertical direction OY has the value:

$$
S_{O Y}=\frac{2 L}{\sqrt{1-\frac{v^{2}}{C^{2} \max }}}
$$

And the distance for the light to travel horizontally OX has the value:

$$
S_{O X}=\frac{2 L}{1-\frac{v^{2}}{C^{2} \max }}
$$

Where:

- L is the arm length of the interferometer

- $v$ is the velocity of the light source

Note: The values of the distances $S_{O X}, S_{O Y}$ are derived from the corresponding time values.

\subsubsection{Analyze the motion direction of the source and light}

According to the law of conservation of time, we have immobility time to change depending on the speed of the object, not depending on the motion directions of the object. Hence we will analyze the motion directions of the object in an example below:

An observer is measuring the distance light travels from position A to position $\mathrm{B}$. Where position $\mathrm{A}$ is considered to be fixed, because although the speed and direction of the source changes, but each light event emitted by the source(an instant moment) is always attached to a fixed point.

$\mathrm{B}$ is the position where the observer has received the light transmitted from position A. But before that the observer is not present at position B, because the observer is moving with the velocity $v_{\text {observer }}$ that he unknown(the earth moving).

$\mathrm{AB}$ distance as measured by observer:

$$
A B=C_{\max } * t_{\text {observer }}
$$


and time $\mathrm{T}$ is calculated according to:

$$
C_{\max } T=A B+v T
$$

Where:

- AB is the distance that light has traveled according to observer's measurement.

- $v$ is the observer's real velocity.

- $C_{\max } T$ is the real distance that the light has traveled.

No one knows the direction of the observer's movement, but immobility time $t_{\text {observer }}$ does not depend on the direction of motion, so we describe the direction of the observer's motion as follows:

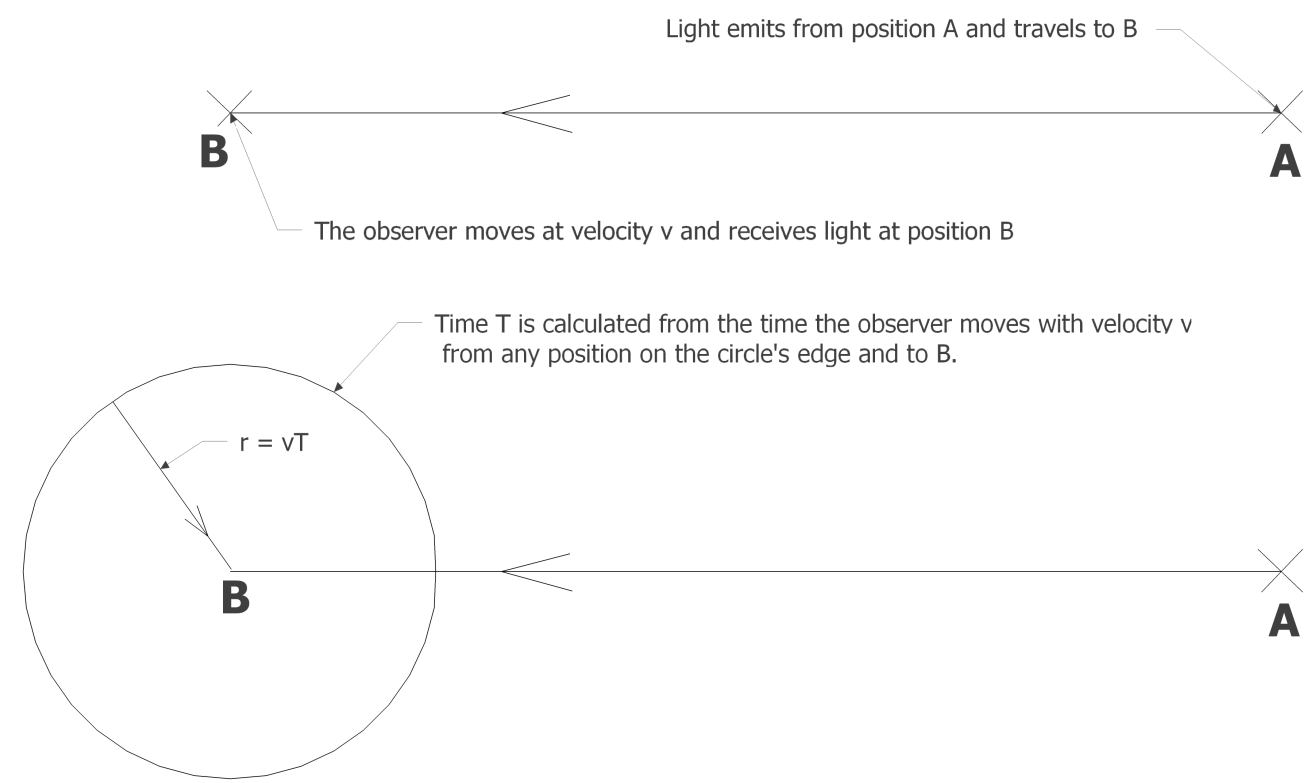

Figure 5: Describe all the motion directions of the observer corresponding to the center circle $B$ with radius $r=v T$

The circle with center $\mathrm{B}$ with radius $r=v T$ represents the distance the observer has traveled since the light was emitted by the source at position A. When light begins to emit from position A then the observer begins to move at velocity $\mathrm{v}$ from any point on the contour of the circle to the center 
position B. Thus, the observer can move in all directions and receive light at position $\mathrm{B}$ for a immobility time $t_{\text {observer }}$

We do not analyze all directions of the observer's motion, we only analyze three directions of motion:

- The direction of motion perpendicular to the direction of motion of light.

- The direction of motion is in the same direction as the direction of motion of light.

- The direction of motion is the opposite of the direction of motion of light.
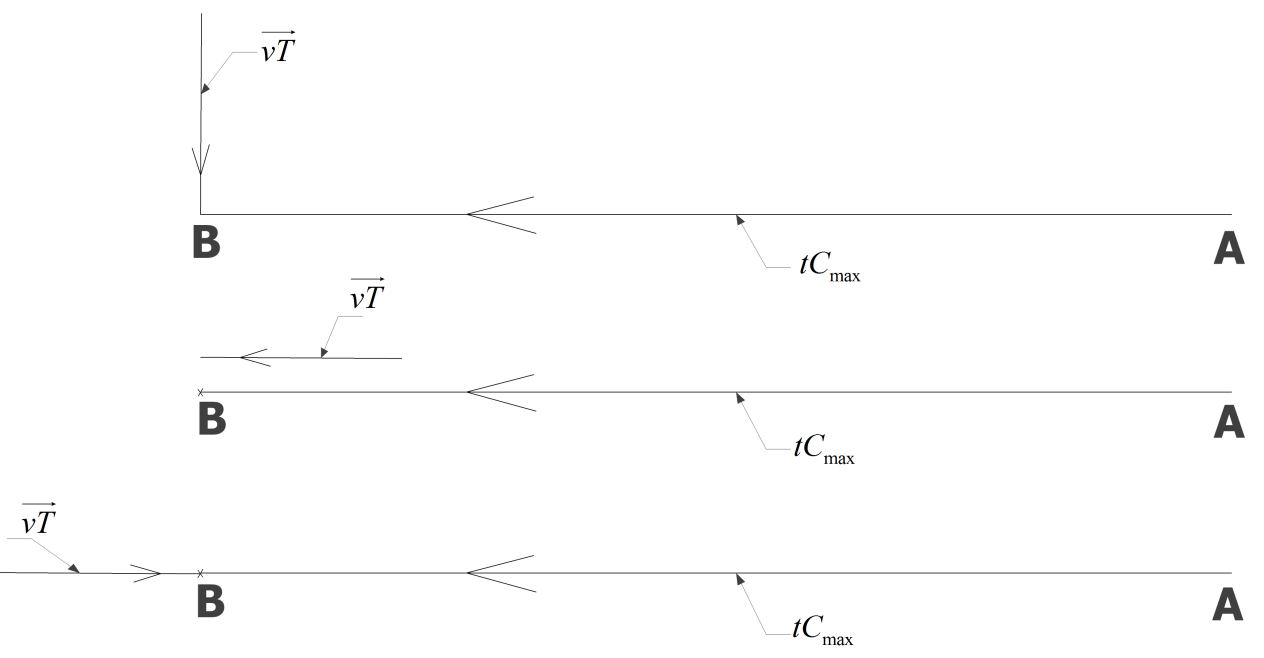

Figure 6: Analyze the three directions of the observer's motion relative to the light's motion direction.

a. Analyze two objects moving in a perpendicular direction.

In this case, we have another method for calculating $\mathrm{T}$ and $C_{\max } T$ values that is to calculate values in a right triangle, we have:

$$
\begin{gathered}
t^{\prime}+t=T \\
\Leftrightarrow t^{\prime} C_{\max }+t C_{\max }=T C_{\max } \\
\Leftrightarrow(\sqrt{v T})^{2}+(\sqrt{A B})^{2}=\left(\sqrt{T C_{\max }}\right)^{2}
\end{gathered}
$$




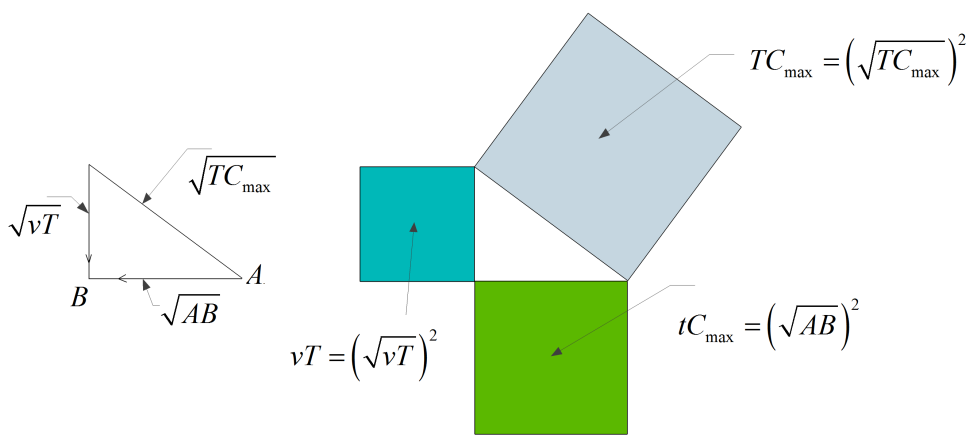

Figure 7: Apply the Pythagorean theorem corresponding to the law of conservation of time.

In there, the values of the sides of the right triangle correspond to $\sqrt{v T}, \sqrt{A B}, \sqrt{T C_{\max }}$. This means that the sides of the right triangle still show the direction of motion perpendicular to the direction of motion of light, but the values $v T, A B, T C_{\max }$ are understood to correspond to the areas:

$$
\begin{gathered}
v T=(\sqrt{v T})^{2} \\
t C_{\max }=(\sqrt{A B})^{2} \\
T C_{\max }=\left(\sqrt{T C_{\max }}\right)^{2}
\end{gathered}
$$

From the above analysis method, we can derive the consequence: Suppose if the observer remains at the original speed and starts moving from position $\mathrm{B}$ (the movement direction is perpendicular to $A B$ ), at the same time light begins to propagate from position $\mathrm{A}$, then the moment the observer and the light meet is $t_{\text {observer }}=\frac{A B}{C_{\max }}$
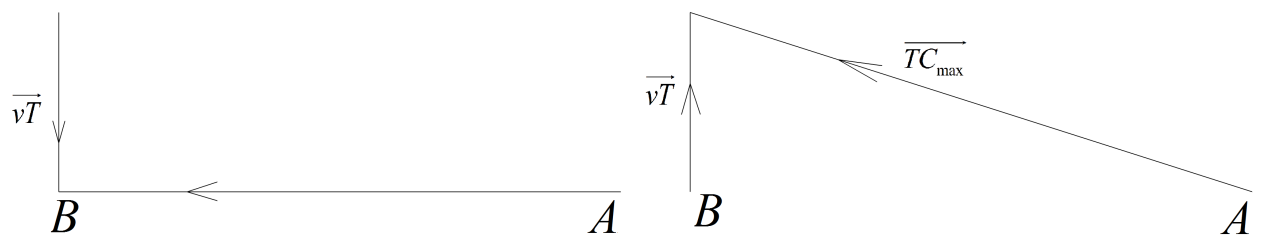
Figure 8: If the observer comes from position B(observer keep the same speed) and the motion direction is perpendicular to $A B$ then the measured distance from $A$ to the light-exposed position is equal to $A B$ value.

Prove this consequence:

Call $T_{2}$ and $t_{2}$ the moment of meeting between light and observer, and applying the law of conservation of time according to the right triangle, we have:

$$
\begin{gathered}
\left(\sqrt{v T_{2}}\right)^{2}+(\sqrt{A B})^{2}=\left(\sqrt{T_{2} C_{\max }}\right)^{2} \\
\rightarrow A B=t_{2} C_{\max } \\
\rightarrow t_{2}=\frac{A B}{C_{\max }}=t_{\text {observer }}
\end{gathered}
$$

Proving end.

Note: We would be wrong if we squared the sides of a right triangle in the form $\left(v T_{2}\right)^{2}+(A B)^{2}=\left(T_{2} C_{\max }\right)^{2}$, because this expression contradicts the law of conservation of time.

b. Analyze two objects moving in the same direction.

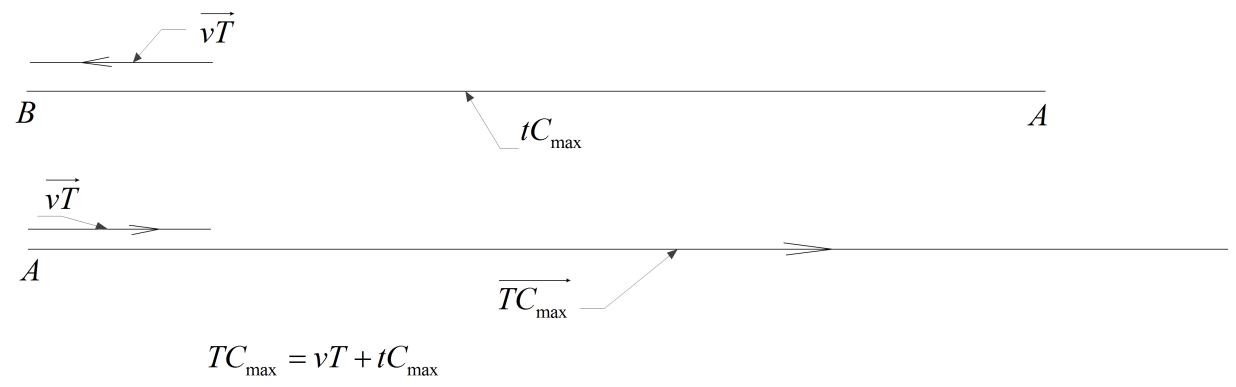

Figure 9: The observer's motion direction coincides with the light's motion direction.

Here is a simple case, there is no other method of calculating $\mathrm{T}$ and $C_{\max } T$ values except for the expression: 


$$
C_{\max } T=A B+v T
$$

In case the observer is also the light emitting source then the two objects will not meet, but the values are still calculated according to the above expression.

c. Analyze two objects moving in opposite directions.

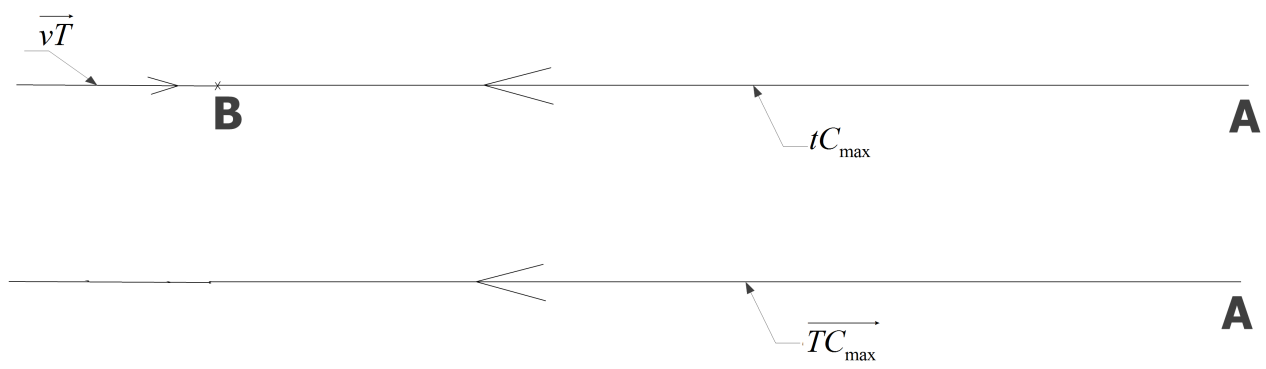

Figure 10: The observer's motion direction in the opposite direction of the motion of light.

This is a special case, we can apply this case to build a problem of finding a given real distance between two objects moving in opposite directions, In which there is an object moving at $C_{\max }$.

Example:

Suppose that the earth and star Proxima Centauri move in opposite directions(towards each other). Thus, we will receive light from Proxima Centauri after a time of 4.2 years. Then, we calculate the distance from Proxima Centauri to earth:

$$
S=t C_{\max } \approx C_{\max } * 1323 * 10^{5}
$$

(The distance $S$ is equivalent to 4.2 light years)

Thus, if we call the initial distance between the earth and Proxima Centauri then the time to meet between the earth and the light emitted by star $A$ is 4.2 years, and $S_{0}$ is calculated by the expression:

$$
\frac{S_{0}}{v T+S}=1
$$


Here, we cannot derive time $T$ because $S$ is calculated according to our immobility time: $S=t C_{\max }$

And:

$$
S_{0}=v T+t C_{\max }
$$

(The result $S_{0}$ satisfies the law of conservation of time)

Through the above example, we see that the meeting time between two objects is not applicable by the expression:

$$
\frac{S}{v T+C_{\max } T}=1 \rightarrow T=\frac{S}{v+C_{\max }}
$$

Because if we apply the expression 6 then it will contradict the law of conservation of time. Also, we cannot determine the real distance $S_{0}$ that the light has traveled, we only have relative distance values.

Therefore, to solve the problem of meeting between two objects with a known relative distance $\left(S=t C_{\max }\right)$, in which one object moves at $C_{\max }$, the time solutions of two objects are calculated by the expression:

$$
\begin{gathered}
t=\frac{S}{C_{\max }} \\
T=t\left(1-\frac{v}{C_{\max }}\right)^{-1}=\frac{S}{C_{\max }-v}
\end{gathered}
$$

Where:

- $t$ is the meeting time corresponding to the immobility time of the object moving with the speed $v, v<C_{\max }$

- $T$ is the common time of two objects, object moving at $C_{\max }$ will not have its immobility time.

Note: If $S$ is calculated by a third object( $S=t_{\text {other_observer }} C_{\max }$, Other object is not the object of the reverse motion problem) then we need to convert the $S$ value to $S_{0}\left(S_{0}=v_{\text {other_observer }} T+t_{\text {other_observer }} C_{\max }\right)$ to solve the problem. 
2.6.3. Michelson-Morley experiment explanation

a. Analysis of light moving in the vertical direction OY.

Call $T_{1}$ the time it takes for the light to travel all the way from the moment it passes through the origin of the semi-silvered mirror until it hits the reflector.

Applying the conservation of time law to calculate the time value $T_{1}$ in a right triangle.:
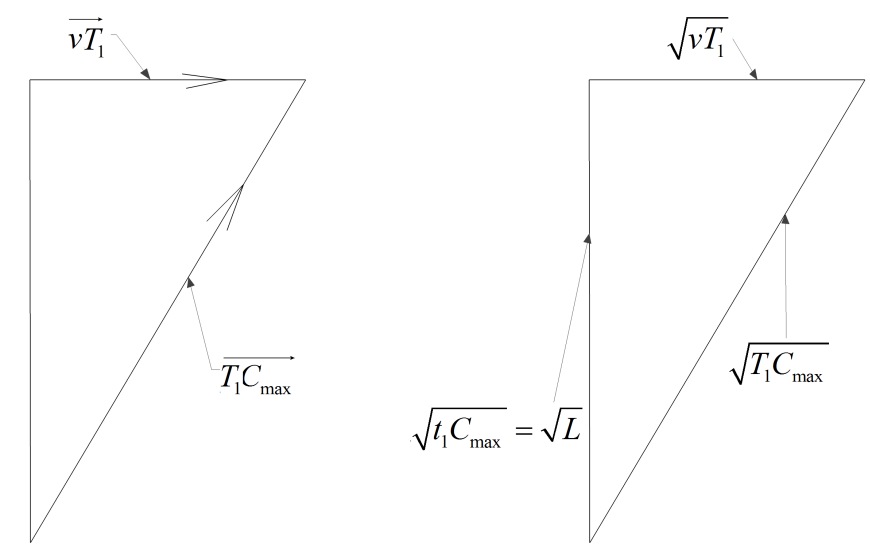

Figure 11: The light travels diagonally corresponding to the hypotenuse of the right triangle.

We have:

$$
\begin{gathered}
(\sqrt{L})^{2}+\left({\sqrt{v T_{1}}}^{2}=\left(\sqrt{C_{\max } T_{1}}\right)^{2}\right. \\
\rightarrow T_{1}=\frac{L}{C_{\max }-v}
\end{gathered}
$$

Call $T_{2}$ the time for the light to travel all the way from the moment it touches the reflector, then moves back to the origin of the half silvered mirror. Thus the distance the light travels corresponding to $T_{2}$ is also a diagonal of the right triangle, so $T_{2}=T_{1}$ 


$$
T_{2}=\frac{L}{C_{\max }-v}
$$

From the values $T_{1}$ and $T_{2}$, we deduce the total time it takes for light to travel the entire distance in the OY vertical direction:

$$
\begin{gathered}
\rightarrow T_{O Y}=T_{1}+T_{2}=\frac{2 L}{C_{\max }-v} \\
\rightarrow t_{O Y}=T_{O Y}\left(1-\frac{v}{C_{\max }}\right)=\frac{2 L}{C_{\max }}
\end{gathered}
$$

Where $t_{O Y}$ is the time that the light source measures when the light has traveled all the way corresponding to time $T_{O Y}, t_{O Y}$ is the immobility time of the light source.

b. Analysis of light moving in the horizontal direction OX.

Call $T_{3}$ the time it takes for the light to travel all the way from the moment it leaves the semi-silvered mirror until it hits the reflector.

$$
\begin{gathered}
C_{\max } T_{3}=L+v T_{3} \\
\rightarrow T_{3}=\frac{L}{C_{\max }-v} \\
\rightarrow t_{3}=\frac{L}{C_{\max }}
\end{gathered}
$$

Call $T_{4}$ the time it takes for the light to travel all the way from the moment it hits the reflector then to the origin of the semi-silver mirror. When the light begins to move in the return reflection direction, the known distance between the light and the source at that moment is valid:

$$
S=C_{\max } T_{3}-v T_{3}=t_{3} C_{\max }
$$

Thus, this is the opposite motion problem as analyzed above, with the given distance $\mathrm{S}$ being a relative value calculated by immobility time $t_{3}$ of the source. Hence we get the values $T_{4}$ and $t_{4}$ according to the expression: 


$$
\begin{gathered}
\rightarrow t_{4}=t_{3}=\frac{L}{C_{\max }} \\
\rightarrow T_{4}=t_{4}\left(1-\frac{v}{C_{\max }}\right)^{-1}=\frac{L}{C_{\max }-v}
\end{gathered}
$$

And we deduce the total time it takes for light to travel the entire distance in the OX horizontal direction:

$$
\begin{gathered}
\rightarrow T_{O X}=T_{3}+T_{4}=\frac{2 L}{C_{\max }-v} \\
\rightarrow t_{O X}=T_{O X}\left(1-\frac{v}{C_{\max }}\right)=\frac{2 L}{C_{\max }}
\end{gathered}
$$

Where $t_{O X}$ is the time that the light source measures when the light has traveled all the way corresponding to time $T_{O X}, t_{O X}$ is the immobility time of the light source.

Comparing the time values, we obtain the following results:

$$
\begin{gathered}
T_{O X}=T_{O Y}=\frac{2 L}{C_{\max }-v} \\
\rightarrow S_{0 \_O X}=S_{0 \_O Y}=\frac{2 L C_{\max }}{C_{\max }-v}
\end{gathered}
$$

And:

$$
\begin{aligned}
& t_{O X}=t_{O Y}=\frac{2 L}{C_{\max }} \\
& \rightarrow S_{O X}=S_{O Y}=2 L
\end{aligned}
$$

Where:

- $S_{0 \_O X}$ and $S_{0 \_O Y}$ are the real distances that light has traveled corresponds to time values $T_{O X}$ and $T_{O Y}$

- $S_{O X}$ and $S_{O Y}$ are the relative distances the light has traveled, calculated according to the immobility time values of the light source $t_{O X}$ and $t_{O Y}$ 
Conclude: Michelson-Morley experiment has been fully explained, and the light has traveled equal distances corresponding to the two directions OX and OY. Therefore, there is no time dilation in the Michelson-Morley experiment, because the relative time change(immobility time) makes us think it is time dilation.

\subsection{Building immobility time relationship between frames of ref- erence}

2.7.1. Building the LCT-transformation

Based on the law of conservation of time, the time of any frame of reference has three components:

$$
t_{\text {immobility }}+t_{\text {motion }}^{\prime}=T_{\text {total }}
$$

Where:

- $t_{\text {immobility }}$ is the immobility time of the frame of reference(relative time), $t_{\text {immobility }}$ is the time displayed on the clock of the frame of reference, the frames of reference with different speeds will have different immobility time.

- $t_{\text {motion }}^{\prime}$ is the motion time of the frame of reference, $t_{\text {motion }}^{\prime}$ is not displayed on the clock of the frame of reference, the frames of reference with different speeds will have different motion time.

- $T_{\text {total }}$ is the total time, $T_{\text {total }}$ is identical and equal in all frames of reference, $T_{\text {total }}$ is not displayed on the clock of the frames of reference.

The immobility time of any frame of reference is calculated according to the expression corresponding to its real velocity:

$$
t_{\text {immobility }}=T_{\text {total }}\left(1-\frac{v}{C_{\max }}\right)
$$

We consider three frames of reference $(\mathrm{A}, \mathrm{B}, \mathrm{C})$ moving in space corresponding to velocities $v_{A}, v_{B}, v_{C}$. Then, the immobility time of the three frames of reference is calculated by:

$$
t_{A}=T\left(1-\frac{v_{A}}{C_{\max }}\right)
$$




$$
\begin{aligned}
& t_{B}=T\left(1-\frac{v_{B}}{C_{\max }}\right) \\
& t_{C}=T\left(1-\frac{v_{C}}{C_{\max }}\right)
\end{aligned}
$$

The immobility time values in the above expressions represents the independence of the three frames of reference. Therefore, the purpose of the time transformation is to build a time relationship between frames of reference to easily make measurements of immobility time and velocity of any frame of reference.

We have:

$$
\frac{t_{B}}{t_{C}}=\frac{C_{\max }-v_{B}}{C_{\max }-v_{C}}
$$

Assuming that we are the frame of reference $\mathrm{A}$ and are observing the motion of two frames of reference $\mathrm{B}$ and $\mathrm{C}$, then we calculate the relative velocities of $\mathrm{B}$ and $\mathrm{C}$ according to $\mathrm{A}$ corresponding to $v_{r e l_{-} B}$ and $v_{r e l_{-} C}$.

Because the relative velocities of $\mathrm{B}$ and $\mathrm{C}$ are $\operatorname{known}\left(v_{r e l_{-} B}\right.$ and $\left.v_{r e l_{C} C}\right)$, we have the relationship between the real velocity and the relative velocity of $\mathrm{B}$ and $\mathrm{C}$ as follows:

$$
v_{B}=v_{A}+\left(1-\frac{v_{A}}{C_{\max }}\right) v_{r e l_{-} B}
$$

and:

$$
v_{C}=v_{A}+\left(1-\frac{v_{A}}{C_{\max }}\right) v_{r e l_{-} C}
$$

On the other hand, we have:

$$
C_{\max }=v_{A}+\left(1-\frac{v_{A}}{C_{\max }}\right) C_{\max }
$$

Therefore: 


$$
C_{\max }-v_{B}=\left(1-\frac{v_{A}}{C_{\max }}\right)\left(C_{\max }-v_{r e l_{\_} B}\right)
$$

and:

$$
C_{\max }-v_{C}=\left(1-\frac{v_{A}}{C_{\max }}\right)\left(C_{\max }-v_{r e l_{-} C}\right)
$$

Finally, we get the result:

$$
\frac{t_{B}}{t_{C}}=\frac{C_{\max }-v_{B}}{C_{\max }-v_{C}}=\frac{C_{\max }-v_{r e l_{-} B}}{C_{\max }-v_{\text {rel_C }}}
$$

Equation 7 is an important equation, it shows the annihilation of time $\mathrm{T}$ and the real velocity of the frames of reference, the physical quantities in the equation are familiar ones and we can easily make measurements. Equation 7 also shows that the immobility time and real velocity of $\mathrm{A}$ (of us) is hidden in the relative velocities $v_{r e l_{-} B}$ and $v_{r e l_{-} C}$, therefore we do not care about our real velocity and immobility time but can still measure the immobility time of $\mathrm{A}$ and $\mathrm{B}$ according to the equation 7 .

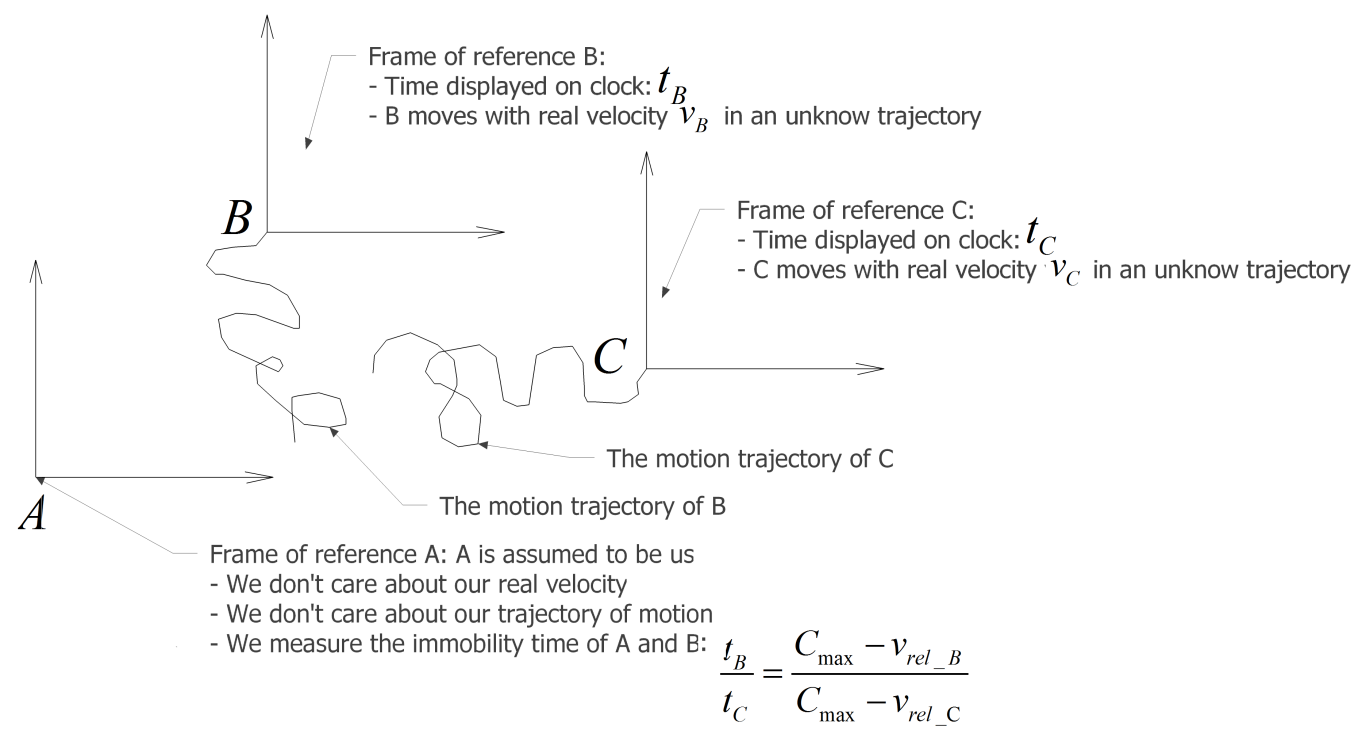


Figure 12: Immobility time relationship between the three frames of reference.

If we only compare time between two frames of reference $\mathrm{A}$ and $\mathrm{B}$ (or $A$ and $C$ ) but based on the relative velocity of $\mathrm{B}$ relative to $\mathrm{A}\left(v_{r e l \_} B\right)$, we have:

$$
\begin{gathered}
t_{B}+t_{B}^{\prime}=t_{A}+t_{A}^{\prime} \\
\Leftrightarrow t_{B}+\left(t_{B}^{\prime}-t_{A}^{\prime}\right)=t_{A} \\
\Leftrightarrow \frac{t_{B}}{t_{A}}+\frac{t_{B}^{\prime}-t_{A}^{\prime}}{t_{A}}=1 \\
\Leftrightarrow \frac{t_{B}}{t_{A}}+\frac{\left(t_{B}^{\prime}-t_{A}^{\prime}\right) C_{\max }}{t_{A}} \frac{1}{C_{\max }}=1 \\
\Leftrightarrow \frac{t_{B}}{t_{A}}=1-\frac{v_{\text {rel_B }}}{C_{\max }}
\end{gathered}
$$

Conclude:

To calculate the immobility time value of frame of reference B, we have 4 methods:

- Calculating immobility time of the frame of reference B directly through total time $\mathrm{T}$, this means not comparing frame of reference $\mathrm{B}$ with any one, or we can understand that B's immobility time(or real velocity) is compared with an absolute frame of reference:

$$
t_{B}=T\left(1-\frac{v_{B}}{C_{\max }}\right)
$$

- If comparing the B-frame with a moving C-frame then the immobility time of $\mathrm{B}$ depends on the real velocity of $\mathrm{C}$ and is calculated by the expression:

$$
\frac{t_{B}}{t_{C}}=\frac{C_{\max }-v_{B}}{C_{\max }-v_{C}}
$$

- If we do not want to calculate the immobility time of the B-frame through the real velocity of $\mathrm{C}$-frame then we can calculate the immobility time of B-frame through the relative velocities $v_{r e l-B}$ and $v_{r e l_{C} C}$ that the A-frame has 
measured:

$$
\frac{t_{B}}{t_{C}}=\frac{C_{\max }-v_{\text {rel_B }}}{C_{\max }-v_{\text {rel_C }}}
$$

- If we only compare time between two frames of reference $\mathrm{A}$ and $\mathrm{B}$ (or $A$ and $C$ ) but based on the relative velocity of $\mathrm{B}$ relative to $\mathrm{A}$, we have:

$$
\frac{t_{B}}{t_{A}}=1-\frac{v_{r e l \_B}}{C_{\max }}
$$

Note:

- Unknown motion trajectory is the trajectory that includes all motion trajectories of the reference system(linear trajectory, circular trajectory, Sineshaped trajectory, ...)

- We call this transformation the LCT-transformation, because it is based on the $\boldsymbol{L}$ aw of $\boldsymbol{C}$ onservation of $\boldsymbol{T}$ ime. The LCT-transformation can replace both the Galilean-transformation and the Lorentz-transformation in physics. Because the LCT-transformation converges all properties of these two transformation(Uniform time $T_{\text {total }}$, time dilation $t_{\text {immobility, independent of the }}$ direction and acceleration of frames of reference,...). In addition, it also has some other properties that the previous two transformations did not have, for example, showing the relationship between the real velocity and the relative velocity of the frames of reference or calculate the time values in motion frames of reference with unknown trajectory.

\subsubsection{Applications of LTC-transformation}

a. Simplify the formula for calculating redshift

In section 2.5, we have the formula 5 for calculating the redshift deviation as follows:

$$
\frac{f_{2}}{f_{1}}=\frac{C_{\max }-v_{\text {source_1 }}}{C_{\max }-v_{\text {source_2 }}}
$$

Where:

- $v_{\text {source_1 }}, v_{\text {source_2 }}$ are the real velocity of a radiation source in two different times 
- $f_{1}, f_{2}$ are radiation frequency of the radiation source in two different times corresponding to velocity $v_{\text {source_1 }}, v_{\text {source_2 }}$

Applying the expression in the LCT-transformation, we have:

$$
\begin{gathered}
\frac{C_{\max }-v_{\text {source_1 }}}{C_{\max }-v_{\text {source_2 }}}=\frac{C_{\max }-v_{\text {rel_source_1 }}}{C_{\max }-v_{\text {rel_source_2 }}} \\
\rightarrow \frac{f_{2}}{f_{1}}=\frac{C_{\max }-v_{\text {rel_source_1 }}}{C_{\max }-v_{\text {rel_source_2 }}}
\end{gathered}
$$

Where:

- $v_{\text {rel_source_1 }}, v_{\text {rel_source_2 }}$ are the relative velocities corresponding to $v_{\text {source_1 }}, v_{\text {source_2 }}$; $v_{\text {rel_source_1 }}, v_{\text {rel_source_2 }}$ are measured by an observer moving at a constant velocity.

b. Solve the problem that Alice calculates Bob's relative velocity

Topic of the problem:

Alice is taking the measurement of Bob's average velocity for the time from $t_{0}$ to $t_{1}$, knowing that Bob is moving with an unknown trajectory. 


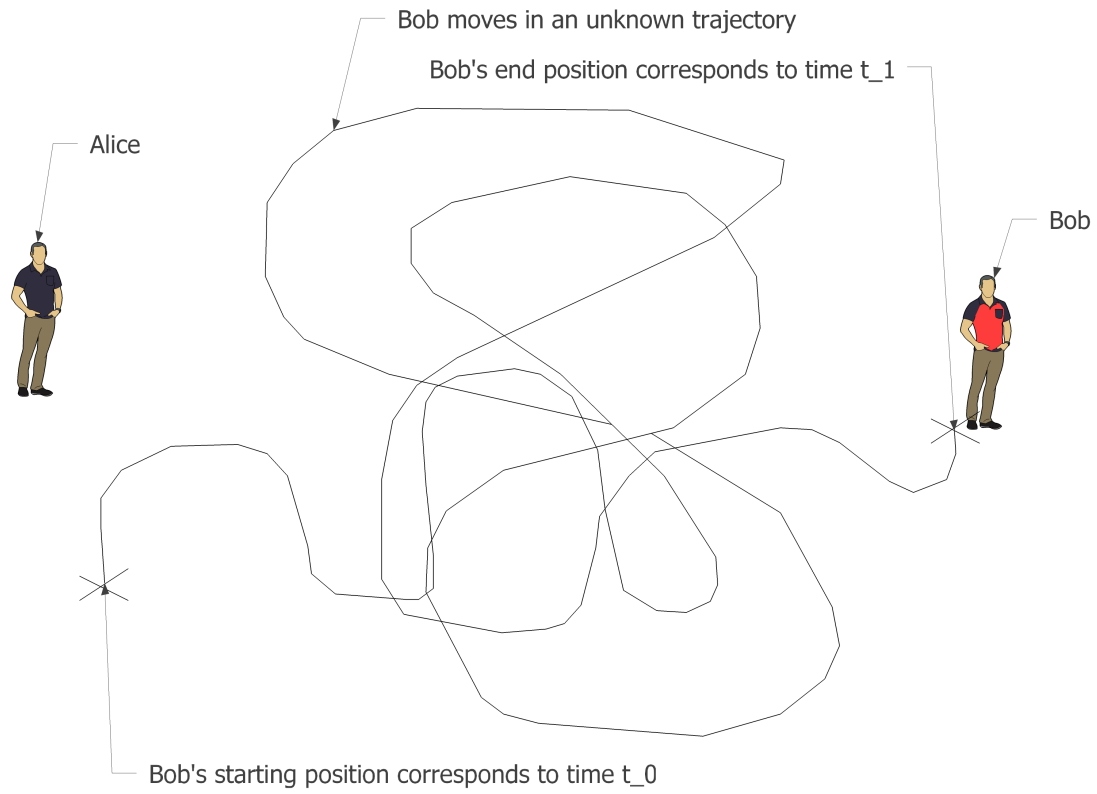

Figure 13: Alice is calculating Bob's relative velocity, while Bob moves with an unknown trajectory.

Solve the problem:

We have the expression for Bob's relative velocity (average velocity in time $\left.\Delta t=t_{1}-t_{0}\right)$ in physics:

$$
v_{r e l \_B o b}=\frac{\Delta s}{\Delta t}=\frac{1}{t_{1}-t_{0}} \int_{t_{0}}^{t_{1}} v(t) d t
$$

Because Bob moves with an unknown trajectory, so Alice cannot construct a functional graph over time, nor can Alice directly measure the value $\Delta s$ to find Bob's relative velocity using equation 12 . Therefore, this is an unsolved problem in physics.

To solve the problem, we apply the formula 11 in the LTC-transformation to calculate the relative velocity:

$$
\frac{t_{\text {Bob }}}{t_{\text {Alice }}}=1-\frac{v_{\text {rel_Bob }}}{C_{\max }}
$$




$$
\rightarrow v_{\text {rel_Bob }}=\left(1-\frac{t_{\text {Bob }}}{t_{\text {Alice }}}\right) C_{\max }
$$

Where:

- $t_{\mathrm{Bob}}$ is Bob's immobility time

- $t_{\text {Alice }}$ is Alice's immobility time

- $v_{\text {rel_Bob }}$ is Bob's relative velocity calculated by Alice's frame of reference

- $C_{\max }$ is the maximum speed in the universe, the speed of light in vacuum

In order to apply the formula, we need to assume that Bob and Alice's clocks have been synchronized before. So after Bob finishes his motion, the time shown on Bob's clock $\left(t_{\text {Bob }}\right)$ will be different from the time shown on Alice's $\operatorname{clock}\left(t_{\text {Alice }}\right)$.

Otherwise, if Bob wants to calculate Alice's relative velocity then the velocity $v_{\text {rel_Alice }}$ will be negative sign, because $t_{\text {Alice }}>t_{\text {Bob }}$ (reflects Bob's real velocity is greater than Alice's real velocity):

$$
v_{\text {rel_Alice }}=\left(1-\frac{t_{\text {Alice }}}{t_{\text {Bob }}}\right) C_{\max }
$$

Note: $\left|v_{\text {rel_Bob }}\right| \neq\left|v_{\text {rel_Alice }}\right| ;\left|\frac{v_{\text {rel_Bob }}}{v_{\text {rel_Alice }}}\right|=\frac{t_{\text {Alice }}}{t_{\text {Bob }}}$

C. The twins paradox problem

Topic of the problem:

There are two twins $\mathrm{A}$ and $\mathrm{B}, \mathrm{A}$ travels space at a very great speed(almost equal to $\left.C_{\max }\right)$ for a long time and then $\mathrm{A}$ returns to meet B. So, after $\mathrm{A}$ meets $\mathrm{B}$, is A older than B or A younger than B?

Solve the problem:

We do not apply the Galilean-transformation to this case, because the Galilean transformation has been replaced by the Lorentz-transformation. But if we apply the Lorentz-transformation to determine that $\mathrm{A}$ is younger than $\mathrm{B}$ then the result will contradict the principle of relativity in special relativity.

Thus, both transformations cannot solve the problem completely. But we can use the LCT-transformation to solve this problem thoroughly: 
Only immobility time $t$ depends on the velocity of the objects. But immobility time $\mathrm{t}$ is just relative time(individual time of each object), it is not a measure to determine the lifespan of objects.

Therefore, the lifespans of A and B will be calculated according to total time $\mathrm{T}$, twins $\mathrm{A}$ and $\mathrm{B}$ have met again while they are still alive so their lifespan is equal up to the moment they $\operatorname{meet}\left(T_{A}=T_{B}=T\right)$. Meanwhile, the time displayed on the clocks of $\mathrm{A}$ and $\mathrm{B}$ will have the difference and the difference value is calculated by the expression:

$$
\frac{t_{A}}{t_{B}}=\frac{C_{\max }-v_{A}}{C_{\max }-v_{B}}
$$

d. Verify the velocity changes of subatomic particles.

Verify that gravity changes the velocity of subatomic particles.

Applying formula 7 in the LTC-transformation, we have:

$$
\frac{C_{\max }-v_{r e l_{-} B}}{C_{\max }-v_{\text {rel_C }}}=\frac{C_{\max }-v_{B}}{C_{\max }-v_{C}}
$$

Thus, if $\mathrm{B}$ and $\mathrm{C}$ are moving at constant speed in space then we get the following result:

$$
\begin{aligned}
& \frac{C_{\max }-v_{B}}{C_{\max }-v_{C}}=\text { const } \\
\rightarrow & \frac{C_{\max }-v_{\text {rel_B }}}{C_{\max }-v_{\text {rel_C }}}=\mathrm{const}
\end{aligned}
$$

Equation 13 shows that if more than one observer takes the measurement of the relative velocities of $\mathrm{B}$ and $\mathrm{C}$ then their proportions remain constant and independent of the velocity of observers:

$$
\rightarrow \frac{C_{\max }-v_{r e l \_B \_o b s e r v e r \_1}}{C_{\max }-v_{\text {rel_C_observer_1 }}}=\frac{C_{\max }-v_{\text {rel_B_observer_2 }}}{C_{\max }-v_{\text {rel_C_observer_2 }}}=\text { const }
$$

Where:

- $v_{\text {rel_B_observer_1 }}, v_{\text {rel_C_observer_1 }}$ are the relative velocities of $B$ and $C$ calculated by observer number 1

- $v_{\text {rel_B_observer_2 }}, v_{\text {rel_C_observer_2 }}$ are the relative velocities of $B$ and $C$ calculated 
by observer number 2

Equation 14 is only correct if the real velocities of $\mathrm{B}$ and $\mathrm{C}$ are constant while two observers measure their relative velocities(two observers have different speeds). Otherwise, if the real velocities of $\mathrm{B}$ and $\mathrm{C}$ change while the observers measure their velocity, we get the result:

$$
\frac{C_{\max }-v_{\text {rel_B_observer_1 }}}{C_{\max }-v_{\text {rel_C_observer_1 }}} \neq \frac{C_{\max }-v_{\text {rel_B_observer_2 }}}{C_{\max }-v_{\text {rel_C_observer_2 }}}
$$

Apply inequality 15 to verify the velocity changes of the particles in the hydrogen atom when placed in two different gravitational conditions. We build the verification method as follows:

There are two hydrogen atoms that have similar properties(the velocities of two electrons are equal, the velocities of two protons are equal) when observer number 1 measured them at the surface of the earth. This is shown in the expression:

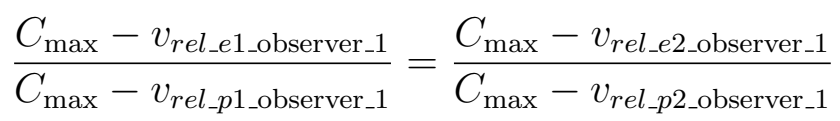

Where:

- $v_{\text {rel_e1_observer_1 }}, v_{\text {rel_p1_observer_1 }}$ are the relative velocities of electron and proton in the hydrogen atom number 1, values were measured by observer number 1

- $v_{\text {rel_e2_observer_1 }}, v_{\text {rel_p2_observer_1 }}$ are the relative velocities of electron and proton in the hydrogen atom number 2 , values were measured by observer number 1

Observer number 2 brings the second hydrogen atom to a height just enough to cause a change in gravity. Then he makes a measurement of the relative velocity of the electron and proton in the second hydrogen atom, the experimental result will return the value:

$$
\frac{C_{\max }-v_{\text {rel_el_observer_1 }}}{C_{\max }-v_{\text {rel_p1_observer_1 }}} \neq \frac{C_{\max }-v_{\text {rel_e2_observer_2 }}}{C_{\max }-v_{\text {rel_p2_observer_2 }}}
$$

Where: 
- $v_{\text {rel_e2_observer_2 }}, v_{\text {rel_p2_observer_2 }}$ are the relative velocities of electron and proton in the hydrogen atom number 2, values were measured by observer number 2

We also note that the change in the velocity of particles is not due to the velocity change of observer number 2 as closely demonstrated above. The only case that leads to the result of inequality 15 is that the relative velocities(or real velocities) of the particles in the hydrogen atom number 2 have changed because they are placed in different gravitational conditions. This also proves that gravity changed the velocity of subatomic particles, leading to the immobility time of subatomic particles changed accordingly.

This verification has important implications, it will open the secrets about black holes and dark matter. For example, if the proof is correct then an atom that approaches the black hole will accelerate and disappear at the event horizon. The disappearance of the atom is due to the fact that the particles in the atom have been moving at $C_{\max }$ for the entire time $T$. This resulted in the atom being unable to emit electromagnetic radiation because it did not exhibit the "disappear - appearance" cycle in time T, at the same time, the atom does not react to electromagnetic radiation because it has disappeared in time $\mathrm{T}$.

But the description of black holes and dark matter based on the law of conservation of time is a distant undertaking, because the next job is to verify the validity of the law of conservation of time and its applications before we can go any further. 


\section{References}

[1] Time - Wikipedia

[2] Atomic clock - Wikipedia

[3] Speed of light - Wikipedia

[4] Quantum tunnelling - Wikipedia

[5] Electron disappears - pubs.acs.org

[6] Velocity - Wikipedia

[7] Metre - Wikipedia

[8] Muon particle - Wikipedia

[9] Redshift - Wikipedia

[10] Radiation - Wikipedia

[11] Time dilation - Wikipedia

[12] Michelson-Morley experiment - Wikipedia 